\title{
ORBIFOLDING FROBENIUS ALGEBRAS
}

\author{
RALPH M. KAUFMANN \\ UNIVERSITY OF SOUTHERN CALIFORNIA, LOS ANGELES, USA*
}

\begin{abstract}
We study the general theory of Frobenius algebras with group actions. These structures arise when one is studying the algebraic structures associated to a geometry stemming from a physical theory with a global finite gauge group, i.e. orbifold theories. In this context, we introduce and axiomatize these algebras. Furthermore, we define geometric cobordism categories whose functors to the category of vector spaces are parameterized by these algebras. The theory is also extended to the graded and super-graded cases. As an application, we consider Frobenius algebras having some additional properties making them more tractable. These properties are present in Frobenius algebras arising as quotients of Jacobian ideal, such as those having their origin in quasi-homogeneous singularities and their symmetries.
\end{abstract}

\section{INTRODUCTION}

The subject of this exposition is the general theory of Frobenius algebras with group actions. These structures arise when one is studying the algebraic structures stemming from a geometry associated to a physical theory with a global finite gauge group [DW,DVVV,IV,V]. The most prominent example of this type in mathematics is the GromovWitten theory of orbifolds $[\mathrm{CR}]$, which are global quotients. The use of orbifold constructions is the cornerstone of the original mirror construction [GP]. The orbifolds under study in that context are so-called Landau-Ginzburg orbifold theories, which have so far not been studied mathematically. These correspond to the Frobenius manifolds coming from singularities and are studied as examples in detail in the present paper.

A common aspect of the physical treatment of quotients by group actions is the appearance of so called twisted sectors. This roughly means that if one wishes to take the quantum version of quotient by a group action, one first has to construct an object for each element in the group together with a group action on this object and in a second step take invariants in all of these components. Whereas classically one

\footnotetext{
* Partially supported by NSF grant DMS \#0070681.
} 
considers only the $G$-invariants of the original object which constitutes the sector associated to the identity, the untwisted sector.

We give the first complete axiomatic treatment, together with a natural geometric interpretation for this procedure, which provides a common basis for applications such as singularities with symmetries or Laundau-Ginzburg orbifolds, orbifold cohomology and quantum cohomology of global quotients and in a sense all other string-orbifold versions of classical theories.

Our treatment shows that the construction of twisted sectors is not merely an auxiliary artifact, but is essential. This is clearly visible in the case of the Frobenius algebra associated to the singularity of type $A_{n}$ together with a $\mathbf{Z} /(n+1) \mathbf{Z}$ action and the singularity of type $A_{2 n-3}$ with $\mathbf{Z} / 2 \mathbf{Z}$ action, which are worked out in detail in the last paragraph. In particular the former example exhibits a version of mirror symmetry in which it is self-dual. The twisted sectors are the key in this mirror duality, since it is the sum of twisted sectors that is dual to the untwisted one.

In the present work, we develop the theory of orbifold Frobenius algebras along the now classical lines of Atiyah, Dubrovin, Dijkgraaf and Segal. I.e. we start by introducing the algebraic structures in an axiomatic fashion. There is an important difference to the theory with trivial group in that there are two structures with slightly different $G$ action to be considered which are nevertheless present. These versions differ by a twist with a character. In the singularity version, on the non-twisted level, they correspond to the different $G$-modules on the cohomology and the Milnor ring [Wa]. In physical terms, these two structures are related by spectral flow. To be even more precise, one structure carries the natural multiplication and the other the natural scalar product. The next step is a cobordism realization of the theory. There are ramifications of the present exposition with [FQ, T], if the above mentioned character is trivial. This is, however, not necessarily the case and in the most interesting examples worked out in the last paragraph, this is not the case.

The key structure here is a non-commutative multiplication on the sum of all the twisted sectors, even before taking invariants. This is a novel approach never considered before.

In order to apply our theory to Landau-Ginzburg models or singularity theory -in a sense the original building blocks for mirror symmetry-, we introduce a large class of examples, so-called special $G$-Frobenius algebras.

This class contains the class of Jacobian $G$-Frobenius algebras which in turn encompass the singularity examples and those of manifolds 
whose cohomology ring can be described as a quotient by a Jacobian ideal. Here it is important to note that everything can be done in a super (i.e. $\mathbf{Z} / 2 \mathbf{Z}$-graded) version. This introduces a new degree of freedom into the construction corresponding to the choice of parity for the twisted sectors. Lastly, we explicitly work out several examples including the transition from the singularity $A_{2 n+3}$ to $D_{n}$ via a quotient by $\mathbf{Z} / 2 \mathbf{Z}$, this is the first purely mathematical version of this correspondence avoiding path integrals. In this situation the presence of the Ramond algebra explains the results obtained by [Wa] in the situation of singularities with symmetries as studied by Arnold. We furthermore show that $* / G$ leads to (twisted)-group algebras.

For Jacobian Frobenius algebras, we also introduce a duality transformation which allows us to show that orbifolding plays the role of mirror symmetry. We show that the pair $\left(A_{n}, A_{1}\right)$ is mirror dual to $\left(A_{1}, A_{n}\right)$ via orbifolding by $\mathbf{Z} /(n+1) \mathbf{Z}$. Applying the same principle to $A_{1} / \mathbf{Z} /(n+1) \mathbf{Z}$ we find the underlying Frobenius algebra structure for the $\mathrm{A}$-model realization of $A_{n}[\mathrm{~W}, \mathrm{JKV}]$.

In the case that the Frobenius algebra one starts out with comes from a semi-simple Frobenius manifold and the quotient of the twisted sector is not trivial, there is unique extension to the level of Frobenius manifolds. This is the case in the above example of $A_{2 n+3}$ and $D_{n}$.

The general theory presented here applies to the orbifold cohomology of global quotients, as worked out in [FG], who found our postulated non-commutative structure in that situation.

Furthermore the theory of special G-twisted Frobenius algebras in the case where $G$ is the symmetric group sheds new light on the construction of [LS], explaining the uniqueness and how the general structure of their multiplication is fixed [K3]. Recently the structure of discrete torsion was uncovered and explained in [K4] to be given by forming tensor products in the sense introduced below with twisted group rings. The synthesis of these results and an application to the Hilbert scheme can be found in [K5].

\section{ACKNOWLEDGEMENTS}

This work is the written version of talk presented at the WAGP2000 conference in October 2000, which contained all algebraic constructions and examples in full detail, and the talk at the workshop on "Mathematical aspects of orbifold string theory" in May 2001, which contained the geometric cobordism part of the third paragraph. I wish to thank the organizers of these conferences, which were immensely stimulating. 
I would also like to thank the Max-Planck Institut für Mathematik in Bonn where much of this work was completed during my stays in 2000 and 2001 as well as the IHES in Bures-sur-Yvette. The research has profited from conversations with Yu. Manin, Y. Ruan, V. Turaev, C. Hertling and B. Penner all of whom it is a pleasure to thank.

Finally, I would like to acknowledge the support from the NSF grant DMS\#0070681.

\section{Frobenius Algebras and Cobordisms}

In this paragraph, we recall the definition of a Frobenius algebra and its relation to the cobordism-category definition of a topological field theory $[\mathrm{A}, \mathrm{Du}, \mathrm{Dij}]$.

\subsection{Frobenius algebras.}

1.1.1. Definition. A Frobenius algebra (FA) over a field $k$ of characteristic 0 is $\langle A, \circ, \eta, 1\rangle$, where

$\mathrm{A}$ is a finite $\operatorname{dim} k$-vector space,

$\circ$ is a multiplication on $A: \circ: A \otimes A \rightarrow A$,

$\eta$ is a non-degenerate bilinear form on $A$, and

1 is a fixed element in $A$ - the unit.

satisfying the following axioms:

a) Associativity

$$
(a \circ b) \circ c=a \circ(b \circ c)
$$

b) Commutativity

$$
a \circ b=b \circ a
$$

c) Unit:

$$
\forall \in a: 1 \circ a=a \circ 1=a
$$

d) Invariance:

$$
\eta(a, b \circ c)=\eta(a \circ b, c)
$$

1.1.2. Remark. By using $\eta$ to identify $A$ and $A^{*}$ - the dual vector space of $A$ - these objects define a one-form $\epsilon \in A^{*}$ called the co-unit and a three-tensor $\mu \in A^{*} \otimes A^{*} \otimes A^{*}$.

Using dualization and invariance these data are interchangeable with $\eta$ and $\circ$ via the following formulas. Explicitly, after fixing a basis $\left(\Delta_{i}\right)_{i \in I}$ of $A$, setting $\eta_{i j}:=\eta\left(\Delta_{i}, \Delta_{j}\right)$ and denoting the inverse metric by $\eta^{i j}$

$$
\begin{aligned}
& \epsilon(a):=\eta(a, 1) \\
& \mu(a, b, c):=\eta(a \circ b, c)=\epsilon(a \circ b \circ c), \\
& a \circ b=\sum_{i j} \mu\left(a, b, \Delta_{i}\right) \eta^{i j} \Delta_{j} \text { and } \\
& \eta(a, b)=\epsilon(a \circ b) .
\end{aligned}
$$


1.1.3. Notation. We call $\rho \in A$ the element dual to $\epsilon$. This is the element which is Poincaré dual to 1 .

1.1.4. Grading. A graded Frobenius algebra is a Frobenius algebra together with a group grading of the vector space $A$ : $A=\bigoplus_{i \in I} A_{i}$ where $I$ is a group together with the following compatibility equations: denote the $I$-degree of an element by deg.

1) 1 is homogeneous; $1 \in A_{d}$ for some $d \in I$

2) $\eta$ is homogeneous of degree $d+D$.

I.e. for homogeneous elements $a, b \eta(a, b)=0$ unless $\operatorname{deg}(a)+$ $\operatorname{deg}(b)=d+D$.

This means that $\epsilon$ and $\rho$ are of degree $D$.

3 ) $\circ$ is of degree $d$, this means that $\mu$ is of degree $2 d+D$, where again this means that

$$
\operatorname{deg}(a \circ b)=\operatorname{deg}(a)+\operatorname{deg}(b)-d
$$

1.1.5. Definition. An even derivation $E \in \operatorname{Der}(A, A)$ of a $G$ twisted Frobenius algebra $A$ is called an Euler field, if it is conformal and is natural w.r.t. the multiplication, i.e. for some $d, D \in k$ it satisfies:

and

$$
\eta(E a, b)+\eta(a, E b)=D \eta(a, b)
$$

$$
E(a b)=E a b+a E b-d a b
$$

Such derivation will define a grading on $A$ by its set of eigenvalues.

1.1.6. Remark. For this type of grading, we will use the group Q. There are two more versions of grading: 1) a $\mathbf{Z} / 2 \mathbf{Z}$ super-grading, which will be discussed in 1.1.13 and 2) a grading by a finite group $G$, which is the content of section 2.1.

1.1.7. Definition. Given an I-graded Frobenius algebra $A$, we define its characteristic series as

$$
\chi_{A}(t):=\sum_{i \in I} \operatorname{dim}\left(A_{i}\right) t^{i}
$$

We refer to the set $\left\{d, D ; i: \operatorname{dim} A_{i} \neq 0\right\}$ as the (I-) spectrum of $A$.

1.1.8. Scaling. If the group indexing the grading has the structure of a $\Lambda$-module, where $\Lambda$ is a ring, we can scale the grading by an element $\lambda \in \Lambda$. We denote the scaled Frobenius algebra by $\lambda A:=\bigoplus_{i \in I} A_{\lambda i}$ where $(\lambda A)_{i}=A_{\lambda i}$

$$
\chi_{\lambda A}(t):=\sum_{i \in I} \operatorname{dim}(\lambda A)_{i} t^{i}=t^{\lambda} \chi_{A}(t)
$$


It is sometimes - but not always- convenient to normalize in such a way that $\operatorname{deg}(1)=0$ where 0 is the unit in $I$. In the case that the grading is given by $\mathbf{Q}$, this means $\operatorname{deg}_{\mathbf{Q}}(1)=0$ and in the finite group case $\operatorname{deg}_{G}(1)=e$ where now $e$ is the unit element of $G$.

1.1.9. Operations. There are two natural operations on Frobenius algebras, the direct sum and the tensor product. Both of these operation extend to the level of Frobenius manifolds, while the generalization of the direct sum is straightforward, the generalization of the tensor product to the level of Frobenius manifolds is quite intricate [K1].

Consider two Frobenius algebras $\mathcal{A}^{\prime}=\left\langle A^{\prime}, \circ^{\prime}, \eta^{\prime}, 1^{\prime}\right\rangle$ and $\mathcal{A}^{\prime \prime}=\left\langle A^{\prime \prime}, \circ^{\prime \prime}\right.$, $\left.\eta^{\prime \prime}, 1^{\prime \prime}\right\rangle$.

1.1.10. Direct sum. Set $\mathcal{A}^{\prime} \oplus \mathcal{A}^{\prime \prime}:=\langle A, \circ, \eta, 1\rangle=\left\langle A^{\prime} \oplus A^{\prime \prime}, \circ^{\prime} \oplus\right.$ $\left.\circ^{\prime \prime}, \eta^{\prime} \oplus \eta^{\prime \prime}, 1^{\prime} \oplus 1^{\prime \prime}\right\rangle$. E.g. $\left(a^{\prime}, a^{\prime \prime}\right) \circ\left(b^{\prime}, b^{\prime \prime}\right)=\left(a^{\prime} \circ^{\prime} b^{\prime}, a^{\prime \prime} \circ^{\prime \prime} b^{\prime \prime}\right)$. The unit is $1=1^{\prime} \oplus 1^{\prime \prime}$ and the co-unit is $\epsilon=\left(\epsilon^{\prime}, \epsilon^{\prime \prime}\right)$.

1.1.11. Lemma. If both Frobenius algebras are graded by the same $I$ then their direct sum inherits a natural grading if and only if the gradings can be scaled s.t.

$$
D^{\prime}+d^{\prime}=D^{\prime \prime}+d^{\prime \prime}:=D+d
$$

where

$$
D=D^{\prime}=D^{\prime \prime}
$$

in this scaling.

Furthermore, the unit will have degree $d^{\prime}=d^{\prime \prime}=d$.

\section{Proof}

The equation (1.5) ensures that the three tensor $\mu$ is homogeneous of degree $D+2 d$. The homogeneity of $\eta$ yields the second condition: for $\eta$ to be homogeneous, we must have that after scaling $\rho^{\prime}$ and $\rho^{\prime \prime}$ are homogeneous of the same degree $D^{\prime}=D^{\prime \prime}=D$. The two equations together imply the homogeneity of $1=\left(1^{\prime}, 1^{\prime \prime}\right)$ of degree $d=d^{\prime}=d^{\prime \prime}$.

1.1.12. Tensor product. Set $\mathcal{A}^{\prime} \otimes \mathcal{A}^{\prime \prime}:=\langle A, \circ, \eta, 1\rangle=\left\langle A^{\prime} \otimes A^{\prime \prime}, \circ^{\prime} \otimes\right.$ $\left.\circ^{\prime \prime}, \eta^{\prime} \otimes \eta^{\prime \prime}, 1^{\prime} \otimes 1^{\prime \prime}\right\rangle$.

E.g. $\left(a^{\prime}, a^{\prime \prime}\right) \circ\left(b^{\prime}, b^{\prime \prime}\right)=\left(a^{\prime} \circ^{\prime} b^{\prime}, a^{\prime \prime} \circ^{\prime \prime} b^{\prime \prime}\right)$. The unit is $1=1^{\prime} \otimes 1^{\prime \prime}$ and the co-unit is $\epsilon=\epsilon^{\prime} \otimes \epsilon^{\prime \prime}$.

There are no conditions for grading. I.e. if both Frobenius algebras are $I$-graded there is a natural induced $I$-grading on their tensor product. The unit is of degree $d=d^{\prime}+d^{\prime \prime}$, the co-unit has degree $D^{\prime}+D^{\prime \prime}$ and the multiplication is homogeneous of degree $d=d^{\prime}+d^{\prime \prime}$. 
1.1.13. Super-grading. For an element $a$ of a super vector space $A=A_{0} \oplus A_{1}$ denote by $\tilde{a}$ its $\mathbf{Z} / 2 \mathbf{Z}$ degree, i.e. $\tilde{a}=0$ if it is even $\left(a \in A_{0}\right)$ and $\tilde{a}=1$ if it is odd $\left(a \in A_{1}\right)$.

1.1.14. Definition. A super Frobenius algebra over a field $k$ of characteristic 0 is $\langle A, \circ, \eta, 1\rangle$, where

$\mathrm{A}$ is a finite $\operatorname{dim} k$-super vector space,

$\circ$ is a multiplication on $A: \circ: A \otimes A \rightarrow A$, which preserves the $\mathbf{Z} / 2 \mathbf{Z}$-grading

$\eta$ is a non-degenerate even bilinear form on $A$, and

1 is a fixed even element in $A_{0}$ - the unit.

satisfying the following axioms:

a) Associativity $(a \circ b) \circ c=a \circ(b \circ c)$

b) Super-commutativity

$$
a \circ b=(-1)^{\tilde{a} \tilde{b}} b \circ a
$$

c) Unit:

$$
\forall \in a: 1 \circ a=a \circ 1=a
$$

d) Invariance:

$$
\eta(a, b \circ c)=\eta(a \circ b, c)
$$

The grading for super Frobenius algebras carries over verbatim.

\subsection{Cobordisms.}

1.2.1. Definition. Let $\mathcal{C O B}$ be the category whose objects are onedimensional closed oriented (topological) manifolds considered up to orientation preserving homeomorphism and whose morphisms are cobordisms of these objects.

I.e. $\Sigma \in \operatorname{Hom}\left(S_{1}, S_{2}\right)$ if $\Sigma$ is an oriented surface with boundary $\partial \Sigma \equiv-S_{1} \coprod S_{2}$.

The composition of morphisms is given by glueing along boundaries with respect to orientation reversing homeomorphisms.

1.2.2. Remark. The operation of disjoint union makes this category into a monoidal category with unit $\emptyset$.

1.2.3. Remark. The objects can be chosen to be represented by disjoint unions of the circle with the natural orientation $S^{1}$ and the circle with opposite orientation $\bar{S}^{1}$. Thus a typical object looks like $S=$ $\coprod_{i \in I} S^{1} \coprod_{j \in J} \bar{S}^{1}$. Two standard morphisms are given by the cylinder, and thrice punctured sphere. 
1.2.4. Definition. Let $\mathcal{V E C \mathcal { T } _ { k }}$ be the monoidal category of finite dimensional $k$-vector spaces with linear morphisms with the tensor product providing a monoidal structure with unit $k$.

1.2.5. Theorem. (Atiyah, Dijkgraaf, Dubrovin) $[\mathrm{A}, \mathrm{Dij}, \mathrm{Du}]$ There is a 1-1 correspondence between Frobenius algebras over $k$ and isomorphism classes of covariant functors of monoidal categories from $\mathcal{C O B}$ to $\mathcal{V} \mathcal{E} \mathcal{T}_{k}$, natural with respect to orientation preserving homeomorphisms of cobordisms and whose value on cylinders $S \times I \in \operatorname{Hom}(S, S)$ is the identity.

Under this identification, the Frobenius algebra $A$ is the image of $S^{1}$, the multiplication or rather $\mu$ is the image of a thrice punctured sphere and the metric is the image of an annulus.

\section{Orbifold Frobenius algebras}

2.1. $G$-Frobenius algebras. We fix a finite group $G$ and denote its unit element by $e$.

2.1.1. Definition. A G-twisted Frobenius algebra (FA) over a field $k$ of characteristic 0 is $\langle G, A, \circ, 1, \eta, \varphi, \chi\rangle$, where

$G$ finite group

$A$ finite $\operatorname{dim} G$-graded $k$-vector space

$A=\oplus_{g \in G} A_{g}$

$A_{e}$ is called the untwisted sector and

the $A_{g}$ for $g \neq e$ are called the twisted sectors.

- a multiplication on $A$ which respects the grading:

$\circ: A_{g} \otimes A_{h} \rightarrow A_{g h}$

1 a fixed element in $A_{e}$-the unit

$\eta$ non-degenerate bilinear form

which respects grading i.e. $\left.g\right|_{A_{g} \otimes A_{h}}=0$ unless $g h=e$.

$\varphi$ an action of $G$ on $A$ (which will be by algebra automorphisms), $\varphi \in \operatorname{Hom}(G, \operatorname{Aut}(A))$, s.t. $\varphi_{g}\left(A_{h}\right) \subset A_{g h g^{-1}}$

$\chi$ a character $\chi \in \operatorname{Hom}\left(G, k^{*}\right)$

Satisfying the following axioms:

Notation: We use a subscript on an element of $A$ to signify that it has homogeneous group degree - e.g. $a_{g}$ means $a_{g} \in A_{g}$ - and we write $\varphi_{g}:=\varphi(g)$ and $\chi_{g}:=\chi(g)$.

a) Associativity

$$
\left(a_{g} \circ a_{h}\right) \circ a_{k}=a_{g} \circ\left(a_{h} \circ a_{k}\right)
$$

b) Twisted commutativity

$$
a_{g} \circ a_{h}=\varphi_{g}\left(a_{h}\right) \circ a_{g}
$$


c) G Invariant Unit:

$$
\begin{aligned}
& 1 \circ a_{g}=a_{g} \circ 1=a_{g} \\
& \text { and } \\
& \varphi_{g}(1)=1
\end{aligned}
$$

d) Invariance of the metric:

$$
\eta\left(a_{g}, a_{h} \circ a_{k}\right)=\eta\left(a_{g} \circ a_{h}, a_{k}\right)
$$

i) Projective self-invariance of the twisted sectors

$$
\varphi_{g} \mid A_{g}=\chi_{g}^{-1} i d
$$

ii) $G$-Invariance of the multiplication

$$
\varphi_{k}\left(a_{g} \circ a_{h}\right)=\varphi_{k}\left(a_{g}\right) \circ \varphi_{k}\left(a_{h}\right)
$$

iii) Projective $G$-invariance of the metric

$$
\varphi_{g}^{*}(\eta)=\chi_{g}^{-2} \eta
$$

iv) Projective trace axiom

$\forall c \in A_{[g, h]}$ and $l_{c}$ left multiplication by $c$ :

$$
\chi_{h} \operatorname{Tr}\left(\left.l_{c} \varphi_{h}\right|_{A_{g}}\right)=\chi_{g^{-1}} \operatorname{Tr}\left(\left.\varphi_{g^{-1}} l_{c}\right|_{A_{h}}\right)
$$

An alternate choice of data is given by a one-form $\epsilon$, the co-unit with $\epsilon \in A_{e}^{*}$ and a three-tensor $\mu \in A^{*} \otimes A^{*} \otimes A^{*}$ which is of group degree $e$, i.e. $\left.\mu\right|_{A_{g} \otimes A_{h} \otimes A_{k}}=0$ unless $g h k=e$.

The relations between $\eta, \circ$ and $\epsilon, \mu$ are analogous to those of 1.1 .2

Again, we denote by $\rho \in A_{e}$ the element dual to $\epsilon \in A_{e}^{*}$ and Poincaré dual to $1 \in A_{e}$.

\subsubsection{Remarks.}

1) $A_{e}$ is central by twisted commutativity and $\left\langle A_{e}, \circ,\left.\eta\right|_{A_{e} \otimes A_{e}}, 1\right\rangle$ is a Frobenius algebra.

2) All $A_{g}$ are $A_{e}$-modules.

3) Notice that $\chi$ satisfies the following equation which completely determines it in terms of $\varphi$. Setting $h=e, c=1$ in axiom iv)

$$
\operatorname{dim} A_{g}=\chi_{g^{-1}} \operatorname{Tr}\left(\left.\varphi_{g}\right|_{A_{e}}\right)
$$

by axiom iii) the action of $\varphi$ on $\rho$ determines $\chi$ up to a sign

$$
\chi_{g}^{-2}=\chi_{g}^{-2} \eta(\rho, 1)=\eta\left(\varphi_{g}(\rho), \varphi_{g}(1)\right)=\eta\left(\varphi_{g}(\rho), 1\right)
$$

4) Axiom iv) forces the $\chi$ to be group homomorphisms, so it would be enough to assume in the data that they are just maps.

2.1.3. Proposition. The $G$ invariants $A^{G}$ of a $G$-Frobenius algebra $A$ form an associative and commutative algebra with unit. This algebra with the induced bilinear form is a Frobenius algebra if and only if $\sum_{g} \chi_{g}^{-2}=|G|$. If $k=\mathbf{C}$ and $\chi \in \operatorname{Hom}(G, U(1))$ this implies $\forall g: \chi_{g}=$ 
Proof. Due to axiom ii) the algebra is associative and commutative. And since 1 is $G$ invariant, the algebra has a unit.

Now suppose $\sum_{g} \chi_{g}^{-2}=|G|$. Then $\left.\eta\right|_{A^{G} \otimes A^{G}}$ is non-degenerate: Let $a \in A^{G}$ and choose $b \in A$ s.t. $\eta(a, b) \neq 0$. Set $\tilde{b}=\frac{1}{|G|} \sum_{g \in G} \varphi_{g}(b) \in A^{G}$. Then

$$
\begin{aligned}
\eta(a, \tilde{b}) & =\frac{1}{|G|} \sum_{g \in G} \eta\left(a, \varphi_{g}(b)\right)=\frac{1}{|G|} \sum_{g \in G} \eta\left(\varphi_{g}(a) \varphi_{g}(b)\right)=\frac{1}{|G|} \sum_{g \in G} \chi_{g}^{-2} \eta(a, b) \\
& =\eta(a, b) \neq 0
\end{aligned}
$$

On the other hand if $\left.\eta\right|_{A^{G} \otimes A^{G}}$ is non-degenerate then let $a, b \in A^{G}$ be such that $\eta(a, b)=1$. It follows:

$1=\eta(a, b)=\frac{1}{|G|} \sum_{g \in G} \eta\left(\varphi_{g}(a), \varphi_{g}(b)\right)=\frac{1}{|G|} \sum_{g \in G} \chi_{g}^{-2} \eta(a, b)=\frac{1}{|G|} \sum_{g \in G} \chi_{g}^{-2}$

so that $\sum_{g} \chi_{g}^{-2}=|G|$.

The last statement follows from the simple fact that since $\forall g \in G$ : $\left|\chi_{g}\right|=1$ and if $\sum_{g} \chi_{g}^{-2}=|G|$ then $\chi_{g}^{-2}=1$ and hence $\chi_{g} \in\{-1,1\}$.

2.1.4. Definition. A $G$-Frobenius algebra is called an orbifold model if the data $\left\langle A^{G}, \circ, 1\right\rangle$ can be augmented by a compatible metric to yield a Frobenius algebra. In this case, we call the Frobenius algebra $A^{G}$ a $G$-orbifold Frobenius algebra.

2.2. Super-grading. We can enlarge the framework by considering super-algebras rather than algebras. This will introduce the standard signs.

2.2.1. Definition. A G-twisted Frobenius super-algebra over a field $k$ of characteristic 0 is $\langle G, A, \circ, 1, \eta, \varphi, \chi\rangle$, where

$G$ finite group

$A$ finite dimensional $\mathbf{Z} / 2 \mathbf{Z} \times G$-graded $k$-vector space $A=A_{0} \oplus A_{1}=\oplus_{g \in G}\left(A_{g, 0} \oplus A_{g, 1}\right)=\oplus_{g \in G} A_{g}$ $A_{e}$ is called the untwisted sector and is even. The $A_{g}$ for $g \neq e$ are called the twisted sectors.

- a multiplication on $A$ which respects both gradings: ० : $A_{g, i} \otimes A_{h, j} \rightarrow A_{g h, i+j}$

1 a fixed element in $A_{e}$-the unit

$\eta$ non-degenerate even bilinear form which respects grading i.e. $\left.g\right|_{A_{g} \otimes A_{h}}=0$ unless $g h=e$.

$\varphi$ an action by even algebra automorphisms of $G$ on $A$, $\varphi \in \operatorname{Hom}_{k-a l g}(G, A)$, s.t. $\varphi_{g}\left(A_{h}\right) \subset A_{g h g^{-1}}$

$\chi \quad$ a character $\chi \in \operatorname{Hom}\left(G, k^{*}\right)$ or if $k=\mathbf{C}, \chi \in \operatorname{Hom}(G, U(1))$ 
Satisfying the axioms a)-d) and i)-iii) of a $G$-Frobenius algebra with the following alteration:

$\left.\mathrm{b}^{\sigma}\right)$ Twisted super-commutativity

$$
a_{g} \circ a_{h}=(-1)^{\tilde{a}_{g} \tilde{a}_{h}} \varphi_{g}\left(a_{h}\right) \circ a_{g}
$$

iv $\left.^{\sigma}\right)$ Projective super-trace axiom

$\forall c \in A_{[g, h]}$ and $l_{c}$ left multiplication by $c$ :

$$
\chi_{h} \operatorname{STr}\left(\left.l_{c} \varphi_{h}\right|_{A_{g}}\right)=\chi_{g^{-1}} \operatorname{STr}\left(\left.\varphi_{g^{-1}} l_{c}\right|_{A_{h}}\right)
$$

where $\mathrm{STr}$ is the super-trace.

\subsubsection{Operations. Restriction.}

If $H \subset G$ and $A=\oplus_{g \in G} A_{g}$ then $\tilde{A}:=\bigoplus_{h \in H} A_{h}$ is naturally a $H$-Frobenius algebra.

Direct Sum. Given a $G$-Frobenius algebra $A$ and an $H$-Frobenius algebra $B$ then $A \oplus B$ is naturally a $G \times H$-Frobenius algebra with the graded pieces $(A \oplus B)_{(g, h)}=A_{g} \oplus B_{h}$.

We define the direct sum of two $G$-Frobenius algebras to be the $G$ Frobenius subalgebra corresponding to the diagonal $\Delta: G \rightarrow G \times G$ in $A \oplus A$.

Tensor product. Given a $G$-Frobenius algebra $A$ and an $H-$ Frobenius algebra $B$ then $\bigoplus_{(g, h)}\left(A_{g} \otimes B_{h}\right)$ is naturally a $G \times H-$ Frobenius algebra $(A \otimes B)_{(g, h)}=A_{g} \otimes B_{h}$.

We define the tensor product of two $G$-Frobenius algebras to be the $G$-Frobenius subalgebra corresponding to the diagonal $G \rightarrow G \times G$ in $A \otimes A$.

Braided Tensor Product. If $A$ and $B$ are two $G$-Frobenius algebras with the same character $\chi$, we can define a braided tensor product structure on $A \otimes B$ by setting $(A \otimes B)_{g}:=\oplus_{k \in G} A_{k} \otimes B_{k^{-1} g}$. For the multiplication we use the sequence

$$
\begin{aligned}
A_{k} \otimes B_{k^{-1} g} \otimes A_{l} \otimes B_{l^{-1} h} \stackrel{\left(i d \otimes i d \otimes \varphi_{k^{-1} g} \otimes i d\right) \circ \tau_{2,3}}{\longrightarrow} & A_{k} \otimes A_{k^{-1} g l g^{-1} k} \otimes B_{k^{-1} g} \otimes B_{l^{-1} h} \\
& \stackrel{\circ \circ}{\rightarrow} A_{g l g^{-1} k} \otimes B_{k^{-1} g l^{-1} h} \quad \text { (2.3) }
\end{aligned}
$$

and $\bigoplus_{k}\left(\varphi_{k} \otimes \varphi_{k h^{-1}}\right)$ for the action of $h$ on $\bigoplus_{k} A_{k} \otimes B_{k^{-1} g}$

2.2.3. Remark. If one thinks in terms of cohomology of spaces the direct sum corresponds to the disjoint union and the tensor product corresponds to the Cartesian product. The origin of the braided tensor product, however, is not clear yet.

2.3. Geometric model - spectral flow. The axioms of the $G$-Frobenius algebra are well suited for taking the quotient, since the multiplication is $G$-invariant. However, this is not the right framework for a geometric 
interpretation. In order to accommodate a more natural coboundary description, we need the following definition which corresponds to the physical notion of Ramond ground states:

2.3.1. Definition. A Ramond $G$-algebra over a field $k$ of characteristic 0 is $<G, V, \bar{o}, v, \bar{\eta}, \bar{\varphi}, \chi>$

$G$ finite group

$V$ finite $\operatorname{dim} G$-graded $k$-vector space

$V=\oplus_{g \in G} V_{g}$

$V_{e}$ is called the untwisted sector and

the $V_{g}$ for $g \neq e$ are called the twisted sectors.

o a multiplication on $V$ which respects the grading:

$\overline{\mathrm{o}}: V_{g} \otimes V_{h} \rightarrow V_{g h}$

$v$ a fixed element in $V_{e}$-the unit

$\bar{\eta}$ non-degenerate bilinear form

which respects grading i.e. $\left.\bar{\eta}\right|_{V_{g} \otimes V_{h}}=0$ unless $g h=e$.

$\bar{\varphi}$ an action by of $G$ on $V$, $\bar{\varphi} \in \operatorname{Hom}(G, \operatorname{Aut}(A))$, s.t. $\bar{\varphi}_{g}\left(V_{h}\right) \subset V_{g h g^{-1}}$

$\chi$ a character $\chi \in \operatorname{Hom}\left(G, k^{*}\right)$

Satisfying the following axioms:

Notation: We use a subscript on an element of $V$ to signify that it has homogeneous group degree - e.g. $v_{g}$ means $v_{g} \in V_{g}$ - and we write $\bar{\varphi}_{g}:=\bar{\varphi}(g)$ and $\chi_{g}:=\chi(g)$.

a) Associativity

$$
\left(v_{g} \overline{\mathrm{o}} v_{h}\right) \overline{\mathrm{o}} v_{k}=v_{g} \overline{\mathrm{o}}\left(v_{h} \overline{\mathrm{o}} v_{k}\right)
$$

b') Projective twisted commutativity

$$
v_{g} \overline{\mathrm{o}} v_{h}=\chi_{g} \bar{\varphi}_{g}\left(v_{h}\right) \overline{\mathrm{o}} v_{g}=\bar{\varphi}_{g}\left(v_{h} \overline{\mathrm{o}} v_{g}\right)
$$

c') Projectively invariant unit:

$$
v \overline{\mathrm{o}} v_{g}=v_{g} \overline{\mathrm{o}} v=v_{g}
$$

and

$$
\bar{\varphi}_{g}(v)=\chi_{g} v
$$

d) Invariance of the metric:

$$
\eta\left(v_{g}, v_{h} \overline{\mathrm{o}} v_{k}\right)=\eta\left(v_{g} \overline{\mathrm{o}} v_{h}, v_{k}\right)
$$

1') Self-invariance of the twisted sectors

$$
\bar{\varphi}_{g} \mid V_{g}=i d
$$

2') Projective $G$-invariance of multiplication

$$
\bar{\varphi}_{k}\left(v_{g} \overline{\bar{\alpha}} v_{h}\right)=\chi_{k} \bar{\varphi}_{k}\left(v_{g}\right) \overline{\bar{\sigma}} \bar{\varphi}_{k}\left(v_{h}\right)
$$

3') $G$-Invariance of metric

$$
\bar{\varphi}_{g}^{*}(\bar{\eta})=\bar{\eta}
$$

4') Trace axiom 
$\forall c \in V_{[g, h]}$ and $l_{c}$ left multiplication by $c$ :

$\operatorname{Tr}\left(\left.l_{c} \circ \bar{\varphi}_{g}\right|_{V_{h}}\right)=\operatorname{Tr}\left(\left.\bar{\varphi}_{h^{-1}} \circ l_{c}\right|_{V_{g}}\right)$

2.3.2. Definition. A state-space for a $G$-Frobenius algebra $A$ is a quadruple $\langle V, v, \bar{\eta}, \bar{\varphi}\rangle$, where

$V$ is a $G$-graded free rank one $A$-module: $V=\bigoplus_{g \in G} V_{g}$,

$v$ is a fixed generator of $V$ - called the vacuum,

$\bar{\eta}$ is non-degenerate bilinear form on $V$

$\bar{\varphi}$ is a linear $G$-action on $V$ fixing $v$ projectively

i.e. $\bar{\varphi}(g)(\operatorname{span}(v)) \subset \operatorname{span}(v)$

such that these structures are compatible with those of $A$ (we denote $\left.\bar{\varphi}_{g}:=\bar{\varphi}(g)\right)$

a) The action of $A$ respects the grading: $A_{g} V_{h} \subset V_{g h}$.

b) $V_{h}$ is a rank one free $A_{h}-$ module and $V_{h}=A_{h} v$.

c) $\bar{\varphi}_{g}(a v)=\varphi_{g}(a) \bar{\varphi}(v): \forall a \in A, v \in V$

d) For $a, b \in A: \bar{\eta}(a v, b v)=\eta(a, b)$

e) $\forall g, h \in G, c \in A_{[g, h]} \forall c \in V_{[g, h]}$ and $l_{c}$ left multiplication by $c$ : $\operatorname{Tr}\left(\left.l_{c} \circ \bar{\varphi}_{g}\right|_{V_{h}}\right)=\operatorname{Tr}\left(\left.\bar{\varphi}_{h^{-1}} \circ l_{c}\right|_{V_{g}}\right)$

2.3.3. Definition. We call two state spaces isomorphic, if there is an $A$-module isomorphism between the two.

Since state spaces are free rank one $A$-modules, it is clear that all automorphisms are rescalings of $v$.

2.4. Proposition. Given a $G$-twisted Frobenius algebra $A$ there is a unique state space up to isomorphism and the form $\bar{\eta}$ is $G$-invariant (i.e. $\left.\bar{\varphi}_{g}^{*}(\bar{\eta})=\bar{\eta}\right)$.

Proof. We start with a free rank one $A$-module $V$ and reconstruct all other data. The $G$-grading on $V$ is uniquely determined from that of $A$ by axiom $b$ ) and this grading satisfies axiom a). Up to isomorphism, we may assume a generator $v \in V$ is fixed, then axiom d) determines $\bar{\eta}$ from $\eta$. We denote the Eigenvalue of $\bar{\varphi}_{g}$ on $v$ by $l_{g}: \bar{\varphi}_{g} v=l_{g} v$. Notice that due to c) $\bar{\varphi}$ is determined by $l_{g}$. Using axioms b), c) and e), we find that $l_{g}=\chi_{g}$, thus fixing the $G$-action $\bar{\varphi}$.

Namely with $c=1$ and $h=e$ in e):

$$
\operatorname{Tr}\left(\left.\bar{\varphi}_{g}\right|_{V_{e}}\right)=l_{g} \operatorname{Tr}\left(\left.\varphi_{g}\right|_{A_{e}}\right)=l_{g} \chi_{g}^{-1} \operatorname{Tr}\left(\left.\varphi_{e}\right|_{A_{g}}\right)=l_{g} \chi_{g}^{-1} \operatorname{Tr}\left(\left.\varphi_{e}\right|_{V_{g}}\right)
$$

The equality $\bar{\varphi}_{g}=\varphi_{g} l_{g}$ implies that $\bar{\eta}$ is $\bar{\varphi}$ invariant:

$$
\bar{\eta}\left(\bar{\varphi}_{g}(a v), \bar{\varphi}_{g}(b v)\right)=l_{g}^{2} \eta\left(\varphi_{g}(a), \varphi_{g}(b)\right)=l_{g}^{2} \chi_{g}^{-2} \eta(a, b)=\bar{\eta}(a v, b v)
$$


In general:

$$
\begin{aligned}
\operatorname{Tr}\left(\left.l_{c} \circ \bar{\varphi}_{g}\right|_{V_{h}}\right) & =l_{g} \operatorname{Tr}\left(\left.l_{c} \circ \varphi_{g}\right|_{A_{h}}\right) \\
& =l_{g} \chi_{g}^{-1} \chi_{h^{-1}} \operatorname{Tr}\left(\left.\varphi_{h^{-1}} \circ l_{c}\right|_{A_{g}}\right) \\
& =l_{g} \chi_{g}^{-1} \chi_{h^{-1}} l_{h^{-1}}^{-1} \operatorname{Tr}\left(\left.\bar{\varphi}_{h^{-1}} \circ l_{c}\right|_{V_{g}}\right) \\
& =\operatorname{Tr}\left(\left.\bar{\varphi}_{h^{-1}} \circ l_{c}\right|_{V_{g}}\right)
\end{aligned}
$$

so that with this choice of $\bar{\varphi}$ axiom e) is satisfied.

2.4.1. Remark. A state space inherits an associative multiplication $\bar{o}$ with unit from $A$ via

$$
\forall a, b \in A:(a v) \overline{\mathrm{o}}(b v):=(a \circ b) v
$$

This multiplication makes it into a $G$-Ramond algebra.

This fact leads us to the following definitions:

2.4.2. Definition. The Ramond space of a $G$-Frobenius algebra $A$ is the state-space given by the $G$-graded vector-space

$$
V:=\oplus_{g} V_{g}:=\bigoplus_{g} A_{g} \otimes k
$$

together with the $G$-action $\bar{\varphi}:=\varphi \otimes \chi$, the induced metric $\bar{g}$ and the induced multiplication $\bar{o}$ and fixed element $v=1 \otimes 1$.

2.4.3. Theorem. There is a one-to-one correspondence between isomorphism classes of $G$-Ramond algebras and $G$-Frobenius algebras.

Proof. The association of a Ramond space to a $G$-Frobenius algebra provides the correspondence. The inverse being the obvious reverse twist by $\chi$.

2.4.4. Remark. In the theory of singularities, the untwisted sector of the Ramond space corresponds to the forms $H^{n-1}\left(V_{\epsilon}, \mathbf{C}\right)$ while the untwisted sector of the $G$-twisted Frobenius algebra corresponds to the Milnor ring [Wa]. These are naturally isomorphic, but have different $G$-module structures. In that situation, one takes the invariants of the Ramond sector, while we will be interested in invariants of the $G-$ Frobenius algebra and not only the untwisted sector (cf. [K5] and see also $\S 7)$.

\section{Bundle Cobordisms, Finite Gauge Groups, ORBIFOlding AND $G$-RAMOND ALGEBRAS}

In this section, we introduce two cobordism categories which correspond to $G$-orbifold Frobenius algebras and Ramond $G$-algebras, respectively. Again $G$ is a fixed finite group. 
3.1. Bundle cobordisms. In all situations, gluing along boundaries will induce the composition and the disjoint union will provide a monoidal structure.

3.1.1. Definition. Let $\mathcal{G B C O B}$ be the category whose objects are principal $G$-bundles over one-dimensional closed oriented (topological) manifolds, pointed over each component of the base space, whose morphisms are cobordisms of these objects (i.e. principal $G$-bundles over oriented surfaces with pointed boundary).

More precisely, $B_{\Sigma} \in \operatorname{Hom}\left(B_{1}, B_{2}\right)$ if $\Sigma$ is an oriented surface with boundary $\partial \Sigma=-S_{1} \coprod S_{2}$ and $B_{\Sigma}$ is a bundle on $\Sigma$ which restricts to $B_{1}$ and $B_{2}$ on the boundary.

The composition of morphisms is given by gluing along boundaries with respect to orientation reversing homeomorphisms on the base and covering bundle isomorphisms which align the base-points.

3.1.2. Remark. The operation of disjoint union makes this category into monoidal category with unit $\emptyset$ formally regarded as a principal $G$ bundle over $\emptyset$.

3.1.3. Remark. Typical objects are bundles $B$ over $S=\coprod_{i \in I} S^{1} \coprod_{j \in J} \bar{S}^{1}$.

Let $\left.\left(S^{1}, \nu\right) \nu \in S^{1}\right)$ be a pointed $S^{1}$.

3.1.4. Structure Lemma. The space $\operatorname{Bun}\left(S^{1}, G\right)$ of $G$ bundles on $\left(S^{1}, \nu\right)$ can be described as follows:

$$
\operatorname{Bun}\left(S^{1}, G\right)=(G \times F) / G
$$

where $F$ is a generic fibre regarded as a principal $G$-space and $G$ acts on itself by conjugation and the quotient is taken by the diagonal action. The space $\operatorname{Bun}^{*}\left(S^{1}, G\right)$ of pointed $G$ bundles on $S^{1}$ is a $G$ bundle over $\operatorname{Bun}\left(S^{1}, G\right)$. Evaluating the monodromy give a projection of $\operatorname{Bun}^{*}\left(S^{1}, G\right)$ onto $G$ whose fiber over $g \in G$ are the centralizers $Z(g)$.

3.1.5. Remark. Usually one uses the identification $\operatorname{Bun}(M, G)=$ $\operatorname{Hom}\left(\pi_{1}(M), G\right) / G$, which we also use in the proof. However, for certain aspects of the theory - more precisely to glues and to include nontrivial characters - it is vital to include a point in the bundle and a trivialization rather then just a point in the base.

Proof of the Structure Lemma. Given a pointed principal $G$ bundle $\left(B, S^{1}, \pi, F, G\right), b \in B$ we set $\nu=\pi(b) \in S^{1}$. The choice of $b \in F=B_{\nu}$ gives an identification of $F$ with $G$, i.e. we let $\beta: G \mapsto F$ be the admissible map in the sense of [St] that satisfies $\beta(e)=b$. We set $g \in G$ to be the element corresponding to the monodromy around 
the generator of $\pi_{1}\left(S^{1}\right)$. Notice that since we fixed an admissible map everything is rigid - there are no automorphisms - and the monodromy is given by an element, not a conjugacy class. Thus we associate to $(B, b)$ the tuple $(g, b)$.

Vice versa, given $(g, b)$ we start with the pointed space $\left(S^{1}, \nu\right)$ and construct the bundle with fiber $G$, monodromy $g$ and marked point $b=\beta(e) \in B_{\nu}$.

The bijectiveness of this construction follows by the classical results quoted above [St].

The choice in this construction corresponds to a choice of a point $b \in B$. Changing $b$ amounts to changing $\beta$ and the monodromy $g$. Moreover, moving the point $\nu=\pi(b)$ and moving $b$ inside the fiber by parallel transport keeps everything fixed. Moving $b$ inside the fixed fiber by translation (once $\nu$ is fixed) corresponds to translation by the group action in the fiber i.e. the translation action of $G$ on $F$ and simultaneous conjugation of the monodromy. Hence, the first claim follows. The second claim follows from the fact that we are dealing with pricipal bundles. Finally evaluating the monodromy of two pointed bundles yields the same result if the bundles are isomorphic and the points are related by a shift in the centralizer of the monodromy.

This observation leads us to the following definition:

3.1.6. Definition. We call a bundle over a closed one-dimensional manifold rigidified if its components are labelled and the bundle is pointed above each component of the base and trivializations around the projection of the marked points to the base are fixed. We denote such a bundle $(B, b)$ where $b \subset B: b=\left(b_{0}, \ldots, b_{n}\right)$ is the set of basepoints for each component.

Furthermore, if a given surface has genus zero we realize it in the plane as a pointed disc with all boundaries being $S^{1}$. We label the outside circle by 0 .

If $\pi$ is the bundle projection, we set $x_{i}:=\pi\left(b_{i}\right)$, we call $b_{0}$ the basepoint, the $B_{x_{i}}$ the special fibers and $B_{x_{0}}$ the initial fiber.

\subsection{Rigidified bundle cobordisms and $G$-Frobenius algebras.}

3.2.1. Definition. Let $\mathcal{G B C O B}{ }^{*}$ be the category whose objects are rigidified principal $G$-bundles over one-dimensional closed oriented (topological) manifolds considered up to pull-back under orientation preserving homeomorphism of the base respecting the markings and whose morphisms are cobordisms of these objects (i.e. principal $G$ bundles over oriented surfaces with boundary together with rigidification on the boundary, a choice of base-point $x_{0} \in \partial \Sigma$ compatible with 
the rigidification and a choice of curves $\Gamma_{i}$ from $x_{0}$ to $x_{i}$ which identify the trivializations via parallel transport, where we used the notation above.).

I.e. objects are bundles $B$ over $S=\coprod_{i \in I} S^{1} \coprod_{j \in J} \bar{S}^{1}$ with base-points on the various components $\bar{b}_{1}=\left(\left.b_{i} \in B\right|_{S_{i}^{1}}: i \in I\right), \bar{b}_{2}=\left(\left.b_{j} \in B\right|_{\bar{S}_{j}^{1}}\right.$ : $j \in J)$ and $B_{\Sigma} \in \operatorname{Hom}\left(\left(B_{1}, \bar{b}_{1}\right),\left(B_{2}, \bar{b}_{2}\right)\right)$ if $\Sigma$ is an oriented surface with boundary considered up to orientation preserving homeomorphism with boundary $\partial \Sigma=-S_{1} \coprod S_{2}$ - again up to homeomorphism - and $B_{\Sigma}$ is a bundle on $\Sigma$ which restricts to $B_{1}$ and $B_{2}$ on the boundary together with rigidification data for the boundary i.e. $\left(\bar{b}_{1}, \bar{b}_{2}\right)$ with $\bar{b}_{1} \subset B_{1}$ and $\bar{b}_{2} \subset B_{2}$. We will call $S_{1}$ the inputs and $S_{2}$ the outputs.

The extra structure of curves and base-point allows us to identify the special fibres with the initial fibre via parallel transport. Thus we can describe the rigidification data in terms of $b_{0}$, and the group elements $g_{i}$ defined via $\Gamma_{i}\left(b_{0}\right)=g_{i} b_{i}$.

The composition of morphisms is given by gluing along boundaries with respect to orientation reversing homeomorphisms of the base and covering bundle isomorphisms identifying the base-points.

3.2.2. Remark. The operation of disjoint union makes this category into a monoidal category with unit $G$ regarded as a bundle over $\emptyset$ with base point $e$.

3.2.3. Construction. We define the pointed bundle $(g, h)$ to be the bundle wich is obtained by glueing $I \times G$ via the identification $(0, e) \sim(1, g)$ and marking the point $(0, h)$, where $I=[0,1]$ the standard interval.

This produces all monoidal generators.

3.2.4. Remark. By the Construction 3.2.3 up to reversing the orientation, we can produce the monoidal generators of objects of $\mathcal{G B C O B} *$ with the objects coming from $G \times G$.

A generating object can thus be depicted by an oriented circle with labels $(g, h)$ where $(g, h) \in G \times G$. We use the notation $(g, h)$ for positively oriented circles and $\overline{(g, h)}$ for negatively oriented circles. We will consider functors $V$ with an involutive property we have that we will have $V(\overline{(g, h)}) \simeq V\left(\left(g^{-1}, h\right)\right)^{*}$ where $*$ denotes the dual. General objects are then just disjoint unions of these, i.e. tuples $\left(g_{i}, h_{i}\right)$. Homomorphisms on the generators are given by the trivial bundle cylinder with different trivializations on both ends represents the natural diagonal action of $G$ by conjugation and translation described in 3.1.4, so that this diagonal $G$-action is realized in terms of cobordisms. 
The natural $G \times G$ action, however, cannot be realized by these cobordisms and we would like to enrich our situation to this case by adding morphisms corresponding to the $G$ action on the trivializations.

3.2.5. Definition. Let $\mathcal{R G B C O B}$ be the category obtained from $\mathcal{G B C O B} *$ by adding the following morphisms. For any $\mathrm{n}$-tuple $\left(k_{1}, \ldots, k_{n}\right)$ : $k_{i} \in G$ and any object $\left(g_{i}, h_{i}\right): i=1, \ldots n$ we set

$$
\tau\left(k_{1}, \ldots, k_{n}\right)\left(g_{i}, h_{i}\right)_{i \in\{1, \ldots n\}}:=\left(g_{i}, k_{i} h_{i}\right)_{i \in\{1, \ldots n\}}
$$

We call these morphisms of type II and the morphisms coming from $\mathcal{G} B C O B *$ of type I. We also sometimes write $I I_{k}$ for $\tau_{k}$.

3.2.6. Remark. There is a natural forgetful functor from $\mathcal{R G B C O B}$ to $\mathcal{G B C O B}$.

Given a character $\chi \in \operatorname{Hom}\left(G, k^{*}\right)$ we can form the fibre-product $G \times_{\chi} k$. This gives a functor $k[G]_{\chi}$ from the category $\mathcal{V E C} \mathcal{T}_{k}$ to $k[G]-$ $\mathcal{M O D}$, the category of $k[G]$-modules.

3.2.7. Definition. A $G_{\chi}$-orbifold theory is a monoidal functor $V$ from $\mathcal{R G B C O B}$ to $k[G]-\mathcal{M O D}$ satisfying the following conditions:

i) The image of $V$ lies in the image of $k[G]_{\chi}$.

ii) Objects of $\mathcal{R G B C O B}$ which differ by morphisms of type II are mapped to the same object in $k[G]-\mathcal{M O D}$.

iii) The value on morphisms of type I does not depend on the choice of connecting curves and associated choice of trivializations or base-point.

iv) The morphisms of type II are mapped to the $G$-action by $\chi$.

v) The functor is natural with respect to morphisms of the type $\tau(k, \ldots, k)$. I.e. $V(\tau(k, \ldots, k) \circ \Sigma)=V\left(\tau_{\text {out }}\left(k^{-1}, \ldots, k^{-1}\right)\right) \circ$ $V(\Sigma) \circ V\left(\tau_{\text {in }}(k, \ldots, k)\right)$; where $\tau_{\text {in }}$ and $\tau_{\text {out }}$ operator on the inputs and outputs respectively.

vi) $V$ associates $i d$ to cylinders $B \times I,(b, 0) \in B \times 0,(b, 1) \in B \times 1$ considered as cobordisms from $(B, b)$ to itself.

vii) $V$ is involutive: $V(\bar{S})=V^{*}(S)$ where $*$ denotes the dual vector space with induced $\mathrm{k}[\mathrm{G}]$-module structure. In accordance, the morphism of type II commute with involution, i.e. they are mapped to the $G$-action by $\chi^{-1}$.

viii) The functor is natural with respect to orientation preserving homeomorphisms of the underlying surface of a cobordism and pull-back of the bundle. 
3.2.8. Corollary. $G_{\chi}$-orbifold theories are homotopy invariant.

Proof. By standard arguments using naturality and vi) a homotopy of an object $S$ induces an identity on its image. More precisely given a homotopy of objects $f_{t}: S \mapsto S$, it induces a map $F: S \times I \mapsto S \times I$ and we have the commutative diagram:

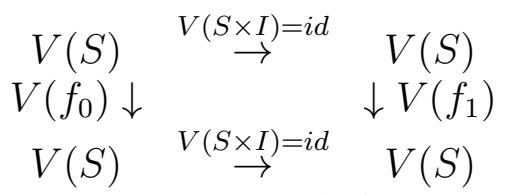

In particular, if $V\left(f_{0}\right)=i d$ then $V\left(f_{1}\right)=i d$.

3.2.9. Remark. Given a choice of connecting curves, we can identify all fibres over special points. Therefore after fixing one identification of a fibre with $G$, we can identify the other marked points as translations of points of parallel transport and label them by group elements, which we will do.

The action of $\tau(k, \ldots, k)$ corresponds to a change of identification for one point and simultaneous re-gauging of all other points via this translation, i.e. a diagonal gauging. Therefore given a cobordism, we can fix an identification of all fibres with $G$.

3.2.10. Definition and Notation. We will fix some standard bundle cobordisms pictured below.

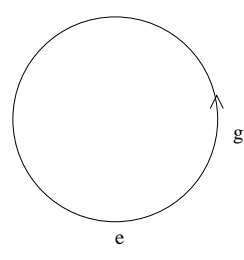

I

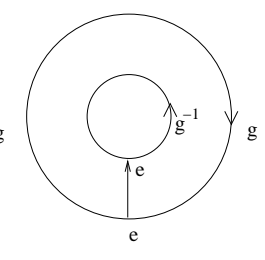

II

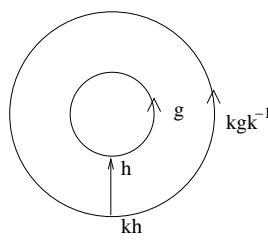

III

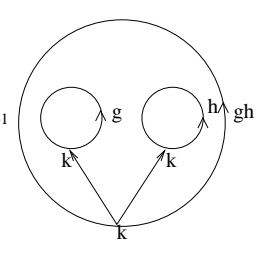

IV

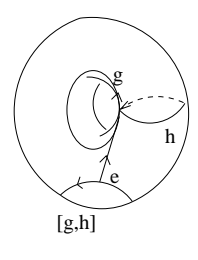

$\mathrm{V}$

I: The standard disc bundle is the disc with a trivial bundle and positively oriented boundary considered as a cobordism between $\emptyset$ and $(e, e)$; it will be denoted by $D$.

II: The standard $g$-cylinder bundle is the cylinder $S^{1} \times I$ with the bundle having monodromy $g$ around $\left(\left(S^{1}, 0\right)\right)$ considered as a cobordism between $(g, e) \coprod \overline{(g, e)}$ and $\emptyset$; it will be denoted by $C_{g}$.

III: The $(g, h)^{k}$-cylinder bundle is the cylinder with a bundle having monodromy $g$ around $\left(S^{1}, 0\right)$ considered as a cobordism between $(g, h)$ and $\left(k g k^{-1}, k h\right)$; it will be denoted by $C_{g, k}^{h}$.

IV: The standard $(g, h)^{k}$-trinion bundle is the trinion with the bundle having monodromies $g$ around the first $S^{1}$ and $h$ around the second $S^{1}$ and translations $e$ for $\tau_{01}, \tau_{02}$ considered as a cobordism between $(g, k),(h, k)$ and $(g h, k)$; it will be denoted by $T_{g, h}^{k}$. 
$\mathrm{V}$ : The $(g, h)$-torus bundle is the once-punctured torus with the principal $G$-bundle having monodromies $g$ and $h$ around the two standard cycles considered as a cobordism between $([g, h], e)$ and $\emptyset$; it will be denoted by $E_{g, h}^{k}$.

3.2.11. Lemma. The bundles over a cylinder that form cobordisms between $(g, e)$ and $(h, k)$ are parameterized by $G$; it is necessary that $g=k h k^{-1}$. These cobordisms are given by the $C_{g, h}^{e}$.

Proof. Given such a bundle over the cylinder $\Sigma_{0}:=S^{1} \times I$ the translation from $B_{\nu, 0}$ to $B_{\nu, 1}$ along $\gamma(t):=(\nu, t)$ is a complete invariant. We fix this element $k \in G$. Since in $\pi_{1}\left(\Sigma_{0},(\nu, 0)\right) C_{1}=\gamma C_{2} \gamma^{-1}$ we must have $g=k h k^{-1}$.

3.2.12. Proposition. To fix a $G_{\chi}$-orbifold theory on the objects of the type $(g, e)$ and to fix a $G_{\chi}$-orbifold theory on the morphisms it suffices to fix it on bundles over the standard cylinder bundle $C$, the $(g, h)$-cylinder bundles $C_{g, h}$, and on the standard trinion $T$.

Proof. The first claim follows from the condition ii) and the monoidal structure. For the second claim first notice that due to the homotopy Lemma 3.2.8 $V$ is fixed on $D$. Also, any bundle over the cylinder is trivial, furthermore by v) we may regard the cylinder as a cobordism from $\left.B\right|_{S^{1}}$ to itself and after applying a morphism of type II we can assume that the boundary objects are of the type $(g, e),(h, k)$. Therefore we know the functor $V$ on all bundles over cylinders. Dualizing and gluing on cylinders, we find that once the functor is defined on the standard trinion, it is defined on all bundles over all trinions. Lastly, given any surface, we can choose a decomposition by a marking into discs, cylinders and trinions, then gluing determines the value of $V$ on this surface.

3.2.13. Proposition and Definition. For any $G_{\chi}$-orbifold theory $V$ set

$$
\begin{array}{ll}
V((g, e)) & =V_{g} \\
V\left(T_{g, h}^{e}\right) & =\bar{\circ}: V_{g} \otimes V_{h} \rightarrow V_{g h} \\
V(D)\left(1_{k}\right) & =v \\
V\left(C_{g}\right) & =\left.\bar{\eta}\right|_{V_{g} \otimes V_{g-1}} \\
V\left(C_{g, h}\right) & =\bar{\varphi}_{h}: V_{g} \mapsto V_{h g h^{-1}}
\end{array}
$$

then this data together with $g$ and $\chi$ form a Ramond $-G$ algebra $\langle G, V, \bar{o}, v, \bar{\eta}, \bar{\varphi}, \chi\rangle$ which we call the associated $G$-Ramond algebra to $V$.

Proof. It is clear by 3.2 .12 that given a $G_{\chi}$ orbifold theory it is completely fixed by its associated $G$-Ramond algebra. The converse is also true: 
The axiom a) follows from the standard gluing procedures of TFT. I.e. the usual gluing of a disc with 3 holes from two discs with two holes in two different ways.
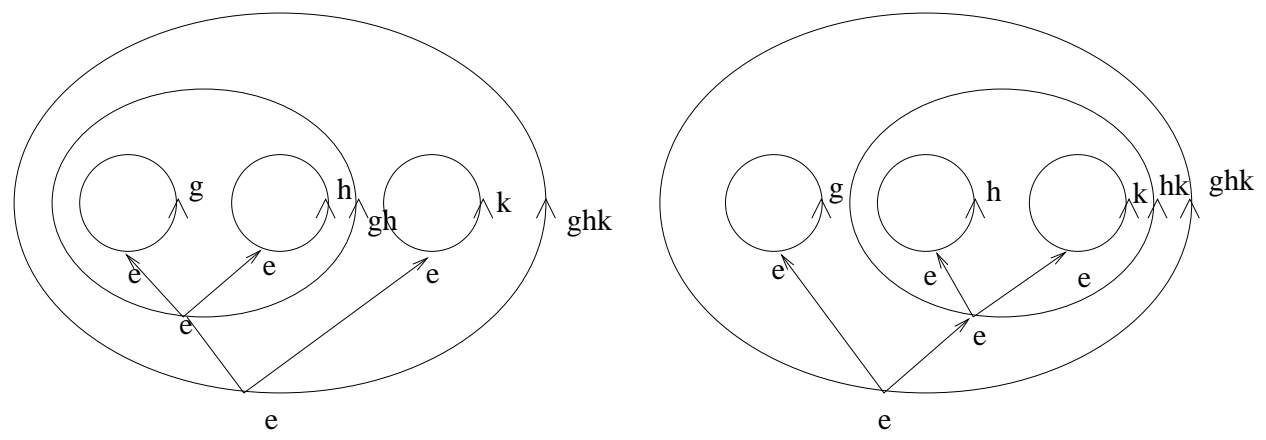

For axiom b') we regard the following commutative diagrams

$$
\begin{array}{cccccccc}
(g, e) \coprod(h, e) \stackrel{\tau_{12}}{\longrightarrow}(h, e) \coprod(g, e) & (h, e) \coprod(g, e) & & (g, e) \coprod(h, e) \\
\downarrow T_{g, h}^{e} & \Rightarrow & \downarrow I I & \Rightarrow & \downarrow I I I & & \downarrow & \downarrow I V \\
(g h, e) & \stackrel{i d}{\longrightarrow} & (g h, e) & & (g h, g) & & (g h, g) \\
& & & & & \\
& & & & \\
V_{g} \otimes V_{h} & \stackrel{\tau_{12}}{\longrightarrow} & V_{h} \otimes V_{g} & \stackrel{i d}{\longrightarrow} & V_{h} \otimes V_{g} & \stackrel{i d}{\longrightarrow} & V_{h} \otimes V_{g} \\
\downarrow \bar{o} & & & & & & \downarrow \bar{\varphi}_{g} \bar{\circ} \\
V_{g h} & \stackrel{i d}{\longrightarrow} & V_{g h} & \stackrel{i d}{\longrightarrow} & V_{g h} & \stackrel{i d}{\longrightarrow} & V_{g h}
\end{array}
$$

where we have used axiom viii) for the first move and axiom iii) for the second and gluing for the last one.

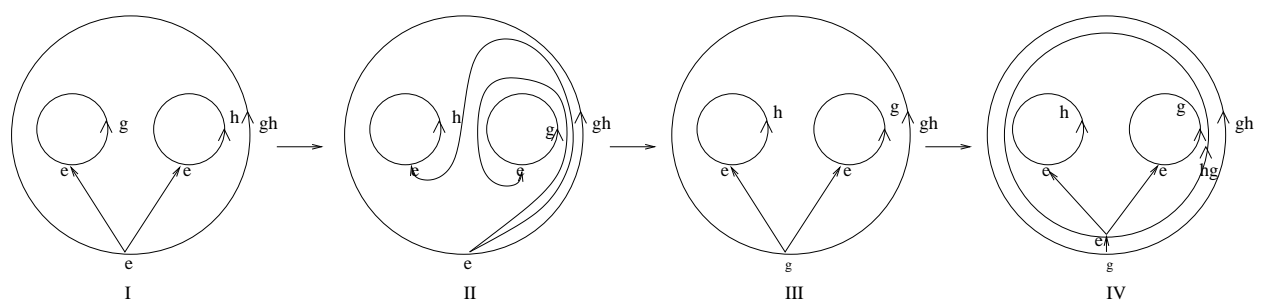

The unit of axiom c) is given by $\bar{D}^{e}$. The projective invariance follows from

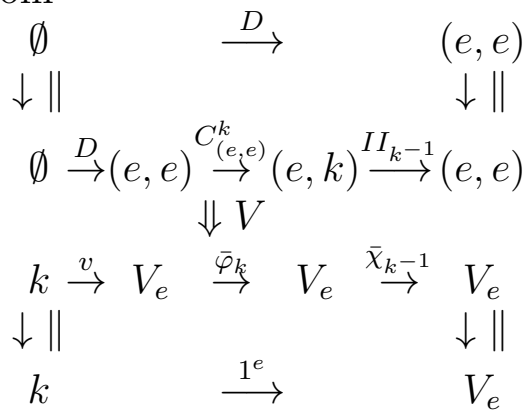

where in the third line $1_{k} \mapsto v \mapsto \chi_{k} v \mapsto v$. 
The axiom d) of the invariance of the metric is again a standard gluing argument.
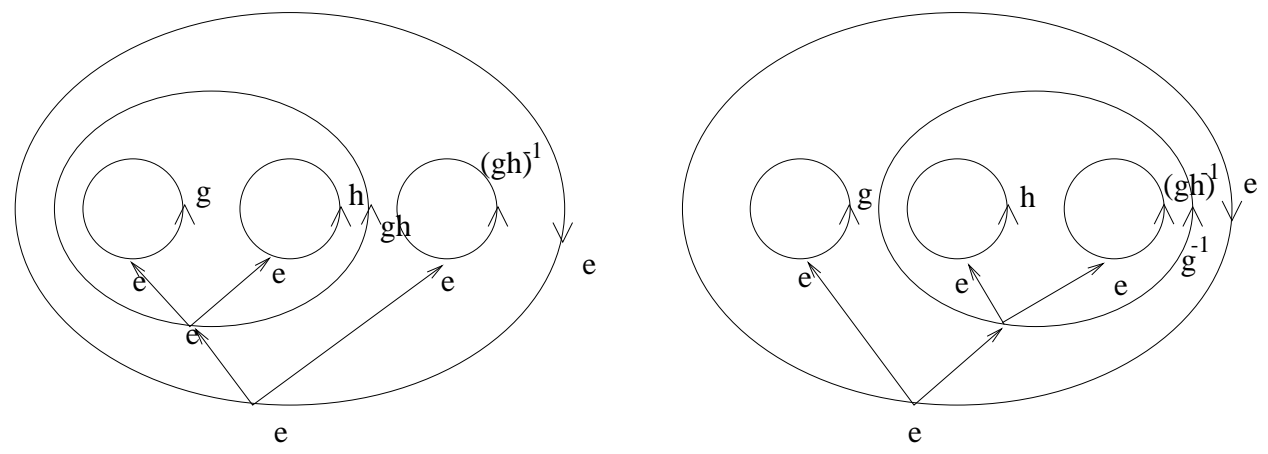

I.e. using $\bar{\epsilon}$ and associativity:

$\bar{\eta}\left(v_{g} \overline{\mathrm{o}} v_{h}, v_{(g h)^{-1}}\right)=\bar{\epsilon}\left(\left(v_{g} \overline{\mathrm{o}} v_{h}\right) \overline{\mathrm{o}} v_{(g h)^{-1}}\right)=\bar{\epsilon}\left(v_{g} \overline{\mathrm{o}}\left(v_{h} \overline{\mathrm{o}} v_{(g h)^{-1}}\right)\right)=\bar{\eta}\left(v_{g}, v_{h} \overline{\mathrm{o}} v_{(g h)^{-1}}\right)$

For axiom 1')

We use the following diagram:

$$
\begin{gathered}
(g, e) \stackrel{i d}{\rightarrow}(g, e) \quad(g, e) \\
\downarrow i d \Rightarrow \downarrow \tilde{C} \Rightarrow \downarrow C \\
(g, e) \stackrel{i d}{\rightarrow}(g, e) \quad(g, g) \\
\Downarrow V \\
V_{g} \stackrel{i d}{\rightarrow} V_{g} \stackrel{i d}{\rightarrow} V_{g} \\
\downarrow i d \\
V_{g} \stackrel{i d}{\rightarrow} V_{g} \stackrel{i d}{\rightarrow} V_{g}
\end{gathered}
$$

where we used axiom viii) for the first move and axiom iii) for the second.
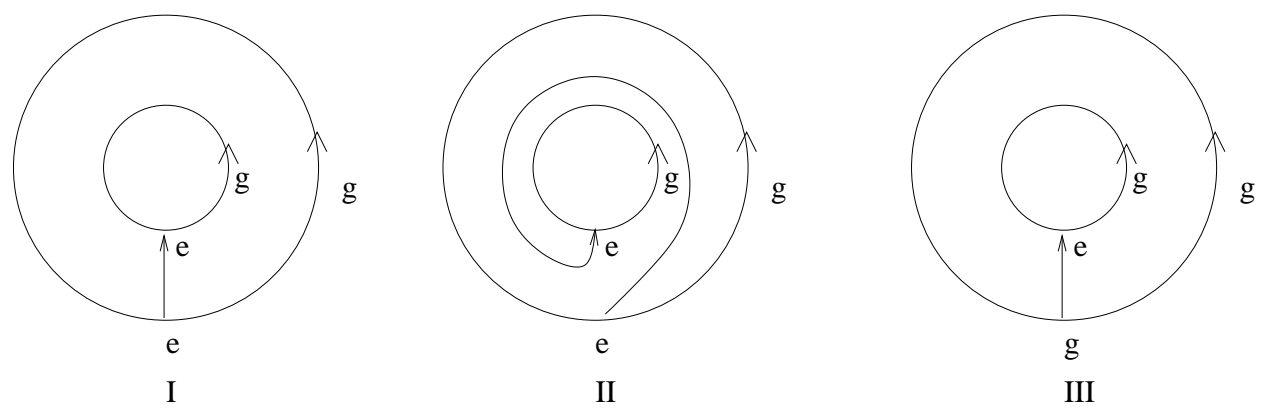

The axiom 2') follows from the diagrams

$$
\begin{aligned}
& (g, e) \coprod_{\downarrow}(h, e) \stackrel{\left(C_{(g, e)}^{k}, C_{(h, e)}^{k}\right)}{\longrightarrow}\left(k g k^{-1}, k\right) \underset{\downarrow}{\coprod}\left(k h k^{-1}, k\right) \stackrel{T_{k g k-1, k h k^{-1}}^{k}}{\longrightarrow}\left(k g h k^{-1}, k\right) \stackrel{\left.C_{(k g h k-1}^{k^{-1}} \longrightarrow k\right)}{\longrightarrow}(g h, e) \\
& V_{g} \otimes V_{h} \quad \stackrel{\bar{\varphi}_{k} \otimes \bar{\varphi}_{k}}{\longrightarrow} \quad V_{k g k^{-1}} \otimes V_{k h k^{-1}} \quad \stackrel{\circ^{k}}{\longrightarrow} \quad V_{k g h k^{-1}} \quad \stackrel{\bar{\varphi}_{k-1}}{\longrightarrow} V_{g h}
\end{aligned}
$$



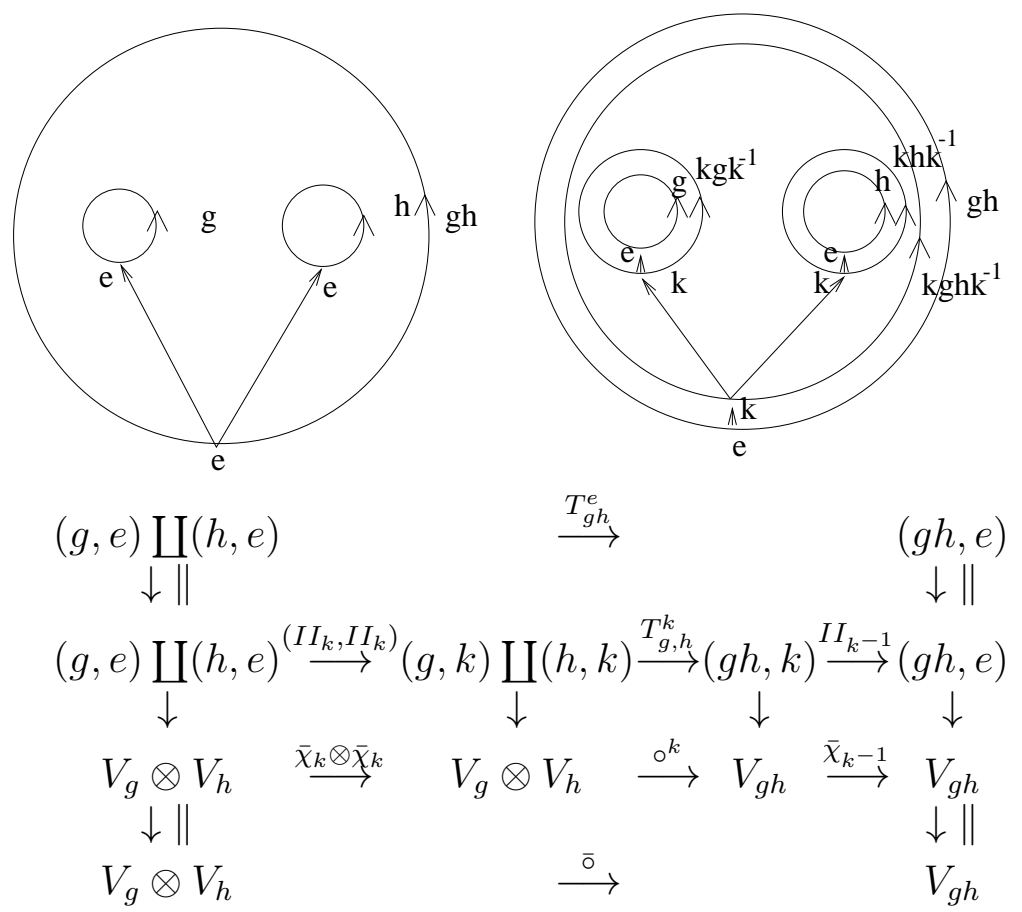

The axiom 3') - G-invariance of the metric - follows from the following diagrams
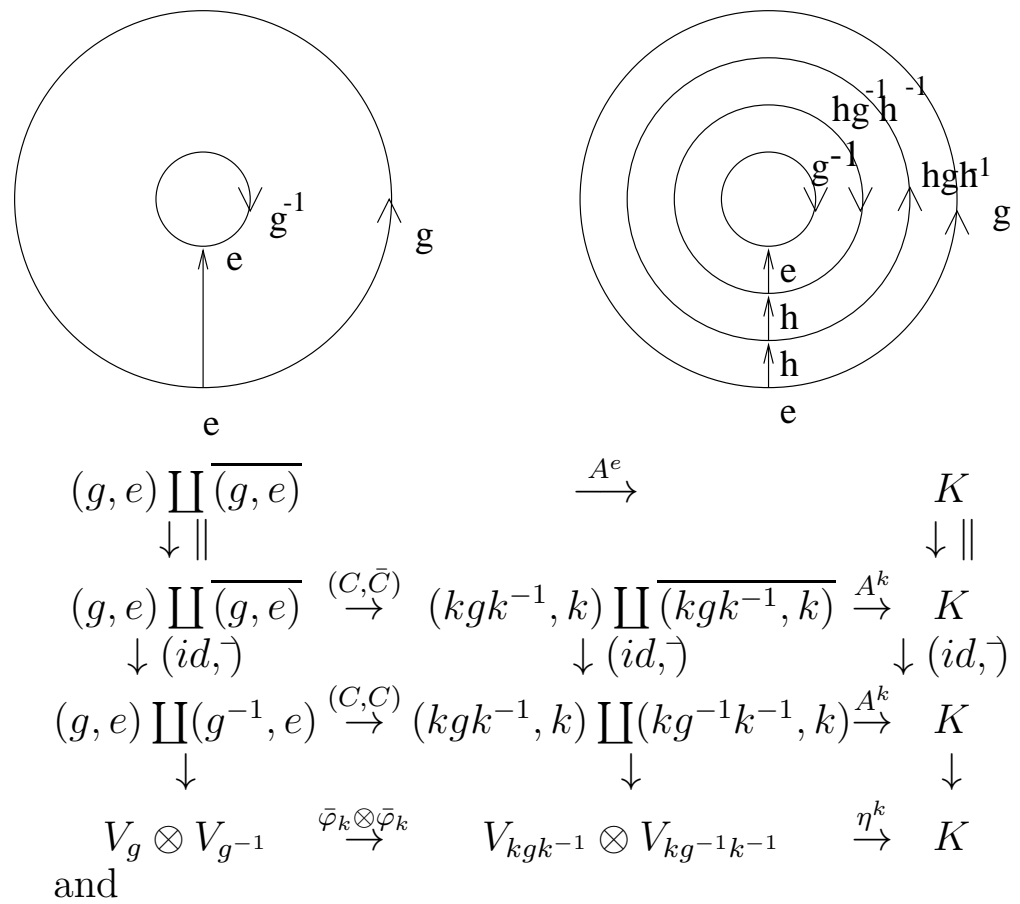


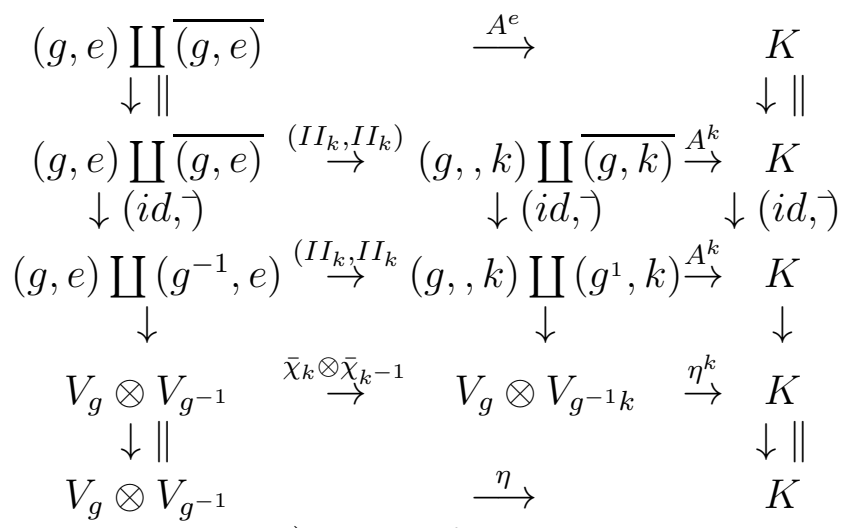

Lastly axiom 4') comes from gluing a once punctured torus in two different ways.

3.2.14. Proposition. Given a $G$-Ramond algebra $V$ there is a unique $G_{\chi}$ orbifold theory $\mathcal{V}$ s.t. $V$ is its associated $G$-Ramond algebra.

Proof.

We need to show that the data is sufficient to reconstruct the functor. For the objects this is clear, due to the monoidal structure. For discs, annuli and trinions the functor is defined by its basic ingredients, glueing annuli $C_{g, h}$ to the basic trinion, and gauging with morphisms of type II. For other morphisms of type I we notice that we can always decompose a surface into trinions, annuli and discs. For each decomposition there are three choices. The choice of a marking for the decomposition, a choice of orientation and a choice of pairs of points over the marked curves. The second and the third choice can be seen to be irrelevant by inserting two annuli $C_{e, k}$ and $A_{k, e}$ with suitable orientation in a normal neighborhood of the curve in question. The first choice is unique up to two operations $[\mathrm{HT}]$ which correspond to associativity and the trace axiom, and is thus also irrelevant.

Combining the Propositions 3.2 .12 and 3.2 .14 with 3.2 .13 we obtain:

3.2.15. Theorem. There is a 1-1 correspondence between isomorphism classes $G$-twisted Frobenius algebras and isomorphism classes of $G_{\chi}-$ orbifold theories as $\chi$ runs through the characters of $G$.

Proof. In the standard way, we make the $G$-twisted Frobenius algebras and the $G_{\chi}$-orbifold theories into categories by introducing the following morphisms. For $G$-twisted Frobenius algebras we use algebra homomorphisms respecting all the additional structures and for $G_{\chi}-$ orbifold theories we use natural transformations among functors. The map of associating a $G$-twisted FA to a $G_{\chi}$-orbifold theory then turns into a full and faithful functor which is by reconstruction surjective on the objects. 
An immediate consequence of this is:

3.2.16. Corollary. There is a $1-1$ correspondence between $G$-orbifold FA and isomorphism classes of monoidal functors which are identity on cylinders and satisfy the involutive property from $\mathcal{G B C O B}$ to $\mathcal{V} E C T$ which lift to $\mathcal{R G} \mathcal{B C O B}$.

3.3. Spectral flow. In the previous paragraph, we chose the geometric version to correspond to the Ramond picture, as is suggested by physics, since we are considering the vacuum states in their Hilbertspaces at the punctures. From physics one expects that by the spectral flow, the vacuum states should bijectively correspond to the chiral algebra. In the current setting the difference only manifests itself in a change of $G$-action given by a twist resulting from the character $\chi$. We can directly produce this $G$-action and thereby the $G$-Frobenius algebra by considering the $G$-action not by $C_{e, k}^{e}$, but by $I I_{k^{-1}} \circ C_{e, k}^{e}$. This statement is proven by re-inspection of the commutative diagrams in the proof of 3.2 .13 .

\section{Special $G$-Frobenius Algebras}

In this section we restrict ourselves to a subclass of $G$-twisted Frobenius algebras. This subclass is large enough to contain all $G$-Frobenius algebras arising from singularities, symmetric products and spaces whose cohomology of the fixed point sets are given by restriction of the comhomology of the ambient space. The restriction allows us to characterize the possible $G$-Frobenius structures for a given collection of Frobenius algebras as underlying data in terms of cohomological data. The restriction we will impose (cyclicity of the twisted sectors) can easily be generalized to more generators; which will render everything matrixvalued.

4.1. Definition. A special $G$-Frobenius algebra is a $G$-twisted Frobenius algebra whose components $A_{g}$ are cyclic $A_{e}$-modules, together with two collections of maps $\left(r_{g}\right),\left(i_{g}\right)$ indexed by $G$ where

$r_{g}: A_{e} \rightarrow A_{g}$ is the map of $A_{e}$-modules induced by multiplication, we write $a_{g}:=r_{g}(a)$. In particular, if one sets $1_{g}:=r_{g}(1)$ we obtain $r_{g}(a)=a 1_{g}$. Notice that it is equivalent to specifying the map $r_{g}$ or generators $1_{g}$ of $A_{g}$ as cyclic $A_{e}$-modules.

$i_{g}: A_{g} \mapsto A_{e}$ are collections of maps s.t. each $i_{g}$ is an injection which splits the exact sequence of $A_{e}$-modules.

$$
0 \longrightarrow I_{g} \longrightarrow A \underset{i_{g}}{\stackrel{r_{g}}{\rightleftarrows}} A_{g} \longrightarrow 0
$$


Here $I_{g} \subset A_{e}$ is the annihilator of $1_{g}$ and thus of $A_{g}$. We denote the concatenation of $i_{g}$ and $r_{g}$ by $\pi_{g}$

$$
\pi_{g}=i_{g} \circ r_{g}: A_{e} \rightarrow A_{e}
$$

and we take the statement that $i_{g}$ is a section of $A_{e}$ modules to mean

$$
i_{g}\left(a_{e} b_{g}\right)=\pi_{g}\left(a_{e}\right) i_{g}\left(b_{g}\right)
$$

Furthermore the following should hold:

$$
\forall g \in G: \varphi_{g}\left(1_{h}\right)=\varphi_{g, h} 1_{g h g^{-1}}
$$

for some $\varphi_{g, h} \in K$ and $\varphi_{g, e}=1$.

4.1.1. Remarks. Notice that

$$
\begin{aligned}
\pi_{g}(a b) & =\pi_{g}(a) \pi_{g}(b) \\
r_{g}(a b) & =\pi_{g}(a) r_{g}(b)
\end{aligned}
$$

In particular, multiplication by $1_{g}$ acts as projection: $a 1_{g}=\pi_{g}(a) 1_{g}$ and $\pi_{g}(1)$ acts as identity on $A_{g}$.

4.1.2. Special super $G$-Frobenius algebra. The super version of special $G$-Frobenius algebras is straightforward. Notice that since each $A_{g}$ is a cyclic $A_{e}$-algebra its parity is fixed to be $(-1)^{\tilde{g}}:=\widetilde{1_{g}}$ times that of $A_{e}$. I.e. $a_{g}=i_{g}\left(a_{g}\right) 1_{g}$ and thus $\widetilde{a_{g}}=\widetilde{i_{g}\left(a_{g}\right)} \widetilde{1_{g}}$. In particular if $A_{e}$ is purely even, $A_{g}$ is purely of degree $\tilde{g}$.

4.2. Proposition. Let $\eta_{g}: i_{g}\left(A_{g}\right) \otimes i_{g}\left(A_{g}\right) \rightarrow K$ be given by the following formula:

$$
\eta_{g}(a, b)=\eta\left(r_{g}(a), r_{g^{-1}}(b)\right)=\epsilon\left(\gamma_{g, g^{-1}} \pi_{g}(a b)\right)
$$

Then $i_{g}\left(A_{g}\right) \subset A_{e}$ together with $\eta_{g}$ and the induced multiplication $a \circ_{g} b=i_{g}\left(a \circ r_{g}(b)\right)=\pi_{g}(a \circ b)$ is a Frobenius sub-algebra. Furthermore $\eta_{g}$ establishes an isomorphism between $i_{g}\left(A_{g}\right)$ and $i_{g^{-1}}\left(A_{g^{-1}}\right)$.

Proof. That the multiplication is commutative and associative can be seen as follows

$$
a \circ_{g} b=i_{g}\left(a b 1_{g}\right)=i_{g}\left(b a 1_{g}\right)=b \circ_{g} c
$$

likewise

$\left(a \circ_{g} b\right) \circ_{g} c=i_{g}\left((a b) c 1_{g}\right)=i_{g}\left(a(b c) 1_{g}\right)=a \circ_{g}\left(b \circ_{g} c\right)$. It is clear that $\eta_{g}$ is an invariant form.

The non-degeneracy follows directly from the non-degeneracy of $\eta$ : fix $a \in i_{g}\left(A_{g}\right)$ and let $a_{g} \in A_{g}$ and $b_{g^{-1}} \in A_{g^{-1}}$ s.t. $i_{g}\left(a_{g}\right)=a$ and $\eta\left(a_{g}, b_{g^{-1}}\right) \neq 0$. Set $b:=i_{g^{-1}}\left(b_{g^{-1}}\right)$ and $\tilde{b}=\pi_{g}(b)$ 
Then

$$
\begin{aligned}
\eta_{g}(a, \tilde{b}) & =\eta\left(a 1_{g}, \tilde{b} 1_{g^{-1}}\right)=\eta\left(\tilde{b} a 1_{g}, 1_{g^{-1}}\right)=\eta\left(b a 1_{g}, 1_{g^{-1}}\right) \\
& =\eta\left(a 1_{g}, b 1_{g^{-1}}\right)=\eta\left(a_{g}, b_{g^{-1}}\right) \neq 0
\end{aligned}
$$

The invariance follows from

$\eta_{g}(a b, c)=\eta\left(r_{g}(a b), r_{g^{-1}}(c)\right)=\eta\left(a b 1_{g}, c 1_{g^{-1}}\right)=\eta\left(a 1_{g}, b c 1_{g^{-1}}\right)=\eta_{g}(a, b c)$

With these two properties, we get a map $i_{g}\left(A_{g}\right) \rightarrow i_{g}\left(A_{g}\right)^{*}$ and $\eta$ gives an isomorphism $i_{g}\left(A_{g}\right)^{*} \rightarrow i_{g^{-1}}\left(A_{g^{-1}}\right)$ via $(a, b) \in A_{g} \otimes A_{g^{-1}} \mapsto$ $\eta\left(a 1_{g}, b 1_{g^{-1}}\right)$.

4.3. Proposition. The following equality holds $\pi_{g^{-1}} \circ \pi_{g}=\pi_{g}$ showing that $\left.\pi_{g^{-1}}\right|_{i_{g}\left(A_{g}\right)}=i d$ identifying $i_{g}\left(A_{g}\right)$ and $i_{g^{-1}}\left(A_{g^{-1}}\right)$.

Proof. We have the following:

$$
\begin{aligned}
\eta\left(\pi_{g^{-1}} \circ \pi_{g}(a) 1_{g}, b_{g^{-1}}\right) & =\eta\left(1_{g}, \pi_{g^{-1}}\left(\pi_{g}(a) i_{g^{-1}}\left(b_{g^{-1}}\right)\right) 1_{g^{-1}}\right) \\
& =\eta\left(\pi_{g}(a) 1_{g}, i_{g^{-1}}\left(b_{g^{-1}}\right) 1_{g^{-1}}\right) \\
& =\eta\left(\pi_{g}(a) 1_{g}, b_{g^{-1}}\right)
\end{aligned}
$$

which proves the statement due to the non-degeneracy of $\eta$.

4.3.1. Remark. We can also pull back $\eta_{g}$ and $\circ_{g}$ to $A_{g}$ which will make $A_{g}$ into a Frobenius algebra and $i_{g}$ into an algebra homomorphism.

More precisely we put:

$$
a_{g} \circ_{g} b_{g}:=r_{g}\left(i_{g}\left(a_{g}\right) i_{g}\left(b_{g}\right)\right)=i_{g}\left(a_{g}\right) b_{g}
$$

and

$$
\eta_{g}\left(a_{g}, b_{g}\right):=\epsilon\left(i_{g}\left(a_{g}\right) i_{g}\left(b_{g}\right) \gamma_{g, g^{-1}}\right)
$$

It then follows that

$$
i_{g}\left(a_{g} \circ_{g} b_{g}\right)=i_{g}\left(a_{g}\right) i_{g}\left(b_{g}\right)
$$

4.4. Definition. Let $A$ be a special $G$-twisted Frobenius algebra $A$. We define a $G$ graded 2 -cocycle with values in $A_{e}$ to be a map $\gamma$ : $G \times G \rightarrow A_{e}$ which satisfies

$$
\gamma_{g, h}:=\gamma(g, h) \in i_{g h}\left(A_{g h}\right)
$$

and

$$
\pi_{g h k}\left(\gamma_{g, h} \gamma_{g h, k}\right)=\pi_{g h k}\left(\gamma_{h, k} \gamma_{g, h k}\right)
$$

We will call such a cocycle associative if also $\forall g, h, k \in G$ :

$$
\begin{aligned}
& \pi_{g h k}\left(\pi_{g h}\left(i_{g}\left(A_{g}\right) i_{h}\left(A_{h}\right)\right) i_{k}\left(A_{k}\right) \gamma_{g, h} \gamma_{g h, k}\right)= \\
& \pi_{g h k}\left(i_{g}\left(A_{g}\right) \pi_{h k}\left(i_{h}\left(A_{h}\right) i_{k}\left(A_{k}\right)\right) \gamma_{h, k} \gamma_{g, h k}\right)
\end{aligned}
$$


and we call a cocycle section independent if $\forall g, h \in G$

$$
\left(I_{g}+I_{h}\right) \gamma_{g, h} \subset I_{g h}
$$

4.5. Remark. Notice that if $g$ is section independent then it follows that the multiplication is independent of the choice of section and the cocycle is automatically associative.

4.6. Definition. A non-abelian $G$ 2-cocycle with values in $k^{*}$ is a map $\varphi: G \times G \rightarrow K$ which satisfies:

$$
\varphi_{g h, k}=\varphi_{g, h k h^{-1}} \varphi_{h, k}
$$

where $\varphi_{g, h}:=\varphi(g, h)$ and

$$
\varphi_{e, g}=\varphi_{g, e}=1
$$

Notice that in the case of a commutative group $G$ this says that the $\varphi_{g, h}$ form a two cocycle with values in $K^{*}$.

Furthermore setting $g=h^{-1}$, we find:

$$
\varphi_{g^{-1}, g h g^{-1}}=\varphi_{g, h}^{-1}
$$

4.7. Proposition. A special $G$-twisted Frobenius algebra $A$ gives rise to an associative $G 2$-cocycle $\gamma$ with values in $A$ and to a non-abelian $G$ 2 -cocycle $\varphi$ with values in $k^{*}$, which satisfy the compatibility equations

and

$$
\varphi_{g, h} \gamma_{g h g^{-1}, g}=\gamma_{g, h}
$$

Proof.

$$
\varphi_{k, g} \varphi_{k, h} \gamma_{k g k^{-1}, k h k^{-1}}=\varphi_{k}\left(\gamma_{g, h}\right) \varphi_{k, g h}
$$

Given a special $G$ twisted Frobenius structure on $A$, we define $\gamma_{g, h} \in$ $i_{g h}\left(A_{g h}\right)$ by

and $\varphi_{g, h} \in k^{*}$ by:

$$
1_{g} 1_{h}=\gamma_{g, h} 1_{g h}
$$

$$
\varphi_{g}\left(1_{h}\right)=\varphi_{g, h} 1_{g h g^{-1}}
$$

By associativity of the multiplication, we find that the $\gamma_{g, h}$ define a graded 2-cocycle with values in $A$.

$\gamma_{g, h} \gamma_{g h, k} 1_{g h k}=\gamma_{g, h} 1_{g h} 1_{k}=\left(1_{g} 1_{h}\right) 1_{k}=1_{g}\left(1_{h} 1_{k}\right)=1_{g} \gamma_{h, k} 1_{h k}=\gamma_{h, k} \gamma_{g, h k} 1_{g h k}$

So that

$$
\pi_{g h k}\left(\gamma_{g, h} \gamma_{g h, k}\right)=\pi_{g h k}\left(\gamma_{h, k} \gamma_{g, h k}\right) .
$$

Redoing the calculation with general elements shows the associativity of the cocycle.

$\varphi$ is a group homomorphism so that 
which yields

$$
\begin{gathered}
\varphi_{g h, k} 1_{g h k h^{-1} g^{-1}}=\varphi_{g h}\left(1_{k}\right)=\varphi_{g}\left(\varphi_{h}\left(1_{k}\right)\right)= \\
\varphi_{g}\left(\varphi_{h, k} 1_{h k h^{-1}}\right)=\varphi_{g, h k h^{-1}} \varphi_{h, k} 1_{g h k h^{-1} g^{-1}}
\end{gathered}
$$

$$
\varphi_{g h, k}=\varphi_{g, h k h^{-1}} \varphi_{h, k}
$$

By $G$-twisted commutativity

$$
\gamma_{g, h} 1_{g h}=1_{g} 1_{h}=\varphi_{g}\left(1_{h}\right) 1_{g}=\varphi_{g, h} 1_{g h g^{-1}} 1_{g}=\varphi_{g, h} \gamma_{g h g^{-1}, g} 1_{g h}
$$

So $\gamma_{g, h}$ and the $\varphi_{g, h}$ satisfy

$$
\varphi_{g, h} \pi_{g h}\left(\gamma_{g h g^{-1}, g}\right)=\pi_{g h} \gamma_{g, h}
$$

$\varphi$ is also an algebra automorphism:

$$
\varphi_{k}\left(1_{g}\right) \varphi_{k}\left(1_{h}\right)=\varphi_{k}\left(1_{g} 1_{h}\right)
$$

Expressed in the $\varphi$ 's and $\gamma$ 's:

$$
\varphi_{k, g} \varphi_{k, h} \gamma_{k g k^{-1}, k h k^{-1}}=\varphi_{k}\left(\gamma_{g, h}\right) \varphi_{k, g h}
$$

which gives a formula for the action of $\varphi$ on the $\gamma_{\mathrm{s}}$.

4.7.1. Corollary. A special $G$-Frobenius algebra gives rise to collection of Frobenius-algebras $\left(A_{g}, \circ_{g}, 1_{g}, \eta_{g}\right)_{g \in G}$ together with a $G$-action on $A_{e}$. A graded $G 2$-cocycle with values in $A_{e}$ and a compatible non-abelian $G 2$-cocycle with values in $k^{*}$. Furthermore:

i) $\eta_{e}\left(\varphi_{g}(a), \varphi_{g}(b)\right)=\chi_{g}^{-2} \eta_{e}(a, b)$

ii) $\eta_{g}\left(a_{g}, b_{g}\right)=\eta\left(i_{g}\left(a_{g}\right) i_{g}\left(b_{g}\right) \gamma_{g, g^{-1}}, 1\right)$

iii) The projective trace axiom $\forall c \in A_{[g, h]}$ and $l_{c}$ left multiplication by $c$ :

$$
\chi_{h} \operatorname{Tr}\left(\left.l_{c} \varphi_{h}\right|_{A_{g}}\right)=\chi_{g^{-1}} \operatorname{Tr}\left(\left.\varphi_{g^{-1}} l_{c}\right|_{A_{h}}\right)
$$

4.8. Proposition. (Reconstruction) Given a Frobenius algebra $\left(A_{e}, \eta_{e}\right)$ and a $G$-action on $A: \varphi: G \times A \rightarrow A$ together with the following data

- Frobenius algebras $\left(A_{g}, \eta_{g} \mid g \in G \backslash\{1\}\right)$.

- Injective algebra homomorphisms $i_{g}: A_{g} \rightarrow A_{e}$ s.t. $i_{g}\left(A_{g}\right)=$ $i_{g^{-1}}\left(A_{g^{-1}}\right)$

- Restriction maps: $r_{g}: A \rightarrow A_{g}$ s.t. $r_{g} \circ i_{g}=i d$.

- A graded associative $G 2$ - cocycle $\gamma$ with values in $A_{e}$ and a compatible non-abelian $G 2$ - $\operatorname{cocycle} \varphi$ with values in $k^{*}$, s.t. $\eta_{g}(a, b)=\eta_{g}\left(\gamma_{g, g^{-1}} i_{g}(a), i_{g}(b)\right)$.

- A group homomorphism $\chi: G \rightarrow k^{*}$ 
such that i)-iii) of 4.7 .1 hold, then there is a unique extension of this set of data to a special $G$-twisted Frobenius algebra, i.e. there is a unique special $G$-twisted Frobenius algebra with these underlying data.

\section{Proof.}

Denote the unit of $A_{e}$ by 1 and the unit of $A_{g}$ by $1_{g}$.

Denote by $\pi_{g}: A_{e} \rightarrow i_{g}\left(A_{g}\right)$ the projection to $A_{g}: \pi_{g}:=i_{g} \circ r_{g}$.

Let the $A_{e}$ module structure on $A_{g}$ be given by

$$
a b_{g}:=r_{g}\left(a i_{g}\left(b_{g}\right)\right)
$$

It is clear that $A_{g}$ is a cyclic $A_{e}$-module generated by $1_{g}$.

The algebra structure is then determined by:

$$
a_{g} b_{h}:=i_{g}\left(a_{g}\right) i_{h}\left(b_{h}\right) \gamma_{g, h} 1_{g h}
$$

This multiplication is associative as can be seen by using the associativity of the cocycle.

The $\varphi$ 's determine the $G$-action by: $\varphi_{g}\left(b_{h}\right):=\varphi_{g}\left(i_{h}\left(b_{h}\right)\right) \varphi_{g, h} 1_{g h g^{-1}}$.

The compatibility and (4.8) guarantee that this is a representation and the $G$ action is indeed an action by algebra automorphisms.

The $G$-twisted commutativity $a_{g} b_{h}=\varphi_{g}\left(b_{h}\right) a_{g}$ also follows from the compatibility.

The form $\eta$ is defined the following way:

$$
\eta\left(a_{g}, h_{g^{-1}}\right):=\eta_{e}\left(i_{g}\left(a_{g}\right) i_{g^{-1}}\left(b_{a^{-1}}\right) \gamma_{g g^{-1}}, 1\right)=\eta_{g}\left(i_{g}\left(a_{g}\right), i_{g^{-1}}\left(b_{a^{-1}}\right)\right)
$$

and

$$
\eta\left(a_{g}, h_{h}\right):=0 \text { if } g h \neq 1
$$

This form is non-degenerate by assumption and it is invariant:

$$
\begin{aligned}
\eta\left(a_{g} b_{h}, d_{(g h)^{-1}}\right) & =\eta_{e}\left(i_{g}\left(a_{g}\right) i_{h}\left(b_{h}\right) i_{h^{-1} g^{-1}}\left(d_{h^{-1} g^{-1}}\right) \gamma_{g h} \gamma_{g h, h^{-1} g^{-1}}, 1\right) \\
& =\eta_{e}\left(i_{g}\left(a_{g}\right) i_{h}\left(b_{h}\right) i_{h^{-1} g^{-1}}\left(d_{h^{-1} g^{-1}}\right) \gamma_{h, h^{-1} g^{-1}} \gamma_{g, g^{-1}}, 1\right) \\
& =\eta\left(g_{g}, b_{h} d_{h^{-1} g^{-1}}\right)
\end{aligned}
$$

4.8.1. Remark. By straightforward calculation we have that the projective trace axiom is equivalent to

$$
\begin{aligned}
& \forall g, h \in G ; c 1_{g h g^{-1} h^{-1}} \in A_{[g, h]}, c \in i_{h g g^{-1} h^{-1}}\left(A_{[g h]}\right) \\
& \chi_{h} \varphi_{h, g} \operatorname{Tr}\left(\left.l_{\gamma_{g h g^{-1} h^{-1}, h h^{-1}} c} \varphi_{h}\right|_{i_{g}\left(A_{g}\right)}\right)= \\
& \chi_{g^{-1}} \varphi_{g^{-1}, g h g^{-1}} \operatorname{Tr}\left(\left.\varphi_{g^{-1}} l_{\left.\gamma_{h g^{-1} h^{-1} g, g^{-1} h g}\right) c}\right|_{i_{h}\left(A_{h}\right)}\right)
\end{aligned}
$$


Notice that in the graded case (see below) this condition only needs to be checked for $\operatorname{deg}\left(\gamma_{g h g^{-1} h^{-1}, h g h^{-1}} c\right) \neq 0$ and $\operatorname{deg}\left(\gamma_{h g^{-1} h^{-1} g, g^{-1} h g} c\right) \neq$ 0 .

Furthermore if $[g, h]=e$ then $\gamma_{g h g^{-1} h^{-1}, h g h^{-1}}=\gamma_{h g^{-1} h^{-1} g, g^{-1} h g}=1_{e}$ and $\varphi_{g^{-1}, g h g^{-1}}=\varphi_{g, h}^{-1}=\varphi_{g^{-1}, h}$.

\subsection{Remarks.}

1) If $A_{g} A_{h} \neq 0$ the compatibility condition (4.7) already determines the $\varphi_{g, h} \in k^{*}$.

2) In particular: $\gamma_{g, g}=0$ unless $\chi_{g}=1$ and if $[g, h]=e$ we have $\varphi_{g, h} \varphi_{h g}=1$ or $\gamma_{g, h}=\gamma_{h, g}=0$.

3) If also $A_{g} A_{h} A_{k} \neq 0$ the elements defined by (4.7) automatically satisfy the conditions of non-abelian 2-cocycles and the condition (4.8) is automatically satisfied.

Proof Without loss of generality, we may assume that $1_{g} 1_{h} 1_{k} \neq 0$ then due to the condition (4.7)

$1_{g} 1_{h} 1_{k}=\left(1_{g} 1_{h}\right) 1_{k}=\varphi_{g h}\left(1_{k}\right)\left(1_{g} 1_{h}\right)=\left(\varphi_{g h}\left(1_{k}\right) 1_{g}\right) 1_{h}=\varphi_{g h, k} 1_{g h k h^{-1} g^{-1}} 1_{g} 1_{h}$ and using associativity, we similarly obtain $1_{g} 1_{h} 1_{k}=1_{g}\left(1_{h} 1_{k}\right)=1_{g} \varphi_{h}\left(1_{k}\right) 1_{h}=\varphi_{g}\left(\varphi_{b, k} 1_{h k h^{-1}}\right) 1_{g} 1_{h}=\varphi_{g, h k h^{-1}} \varphi_{h, k} 1_{g h k h^{-1} g^{-1}} 1_{g} 1_{h}$

For the first statement in 2) one just needs to plug $g=h$ into (4.7) and for the second one has to apply the formula twice. For 3) notice that $1_{k} 1_{g} 1_{h}=1_{k}\left(1_{g} 1_{h}\right)=\varphi_{k}\left(1_{g} 1_{h}\right) 1_{k}$ and on the other hand $\left.1_{k} 1_{g} 1_{h}=\varphi_{k}\left(1_{g}\right) 1_{k} 1_{h}\right)=\varphi_{k}\left(1_{g}\right) \varphi_{k}\left(1_{h}\right) 1_{k}$.

A useful technical Lemma to show that $\gamma_{g, h} \neq 0$ is the following

4.10. Lemma. If $\gamma_{g, h}=0$ then $\pi_{h}\left(\gamma_{g, g^{-1}}\right)=0$ and $\pi_{g}\left(\gamma_{h, h^{-1}}\right)=0$

Proof.

If $\gamma_{g, h}=0$ then

$\left.0=\pi_{h}\left(\gamma_{g^{-1}, g h} \gamma_{g, h}\right)\right)=\pi_{h}\left(\gamma_{g^{-1}, g} \gamma_{e, h}\right)=\pi_{h}\left(\gamma_{g^{-1}, g}\right)=\pi_{h}\left(\gamma_{g, g^{-1}}\right)$

and also

$\left.0=\pi_{g}\left(\gamma_{g, h} \gamma_{g h, h^{-1}}\right)\right)=\pi_{g}\left(\gamma_{g, e} \gamma_{h^{-1}, h}\right)=\pi_{g}\left(\gamma_{h, h}^{-1}\right)$

4.10.1. Graded special $G$-Frobenius algebras. Consider a set of graded Frobenius algebras satisfying the reconstruction data: $\left\{\left(A_{g}, \eta_{g}\right)\right.$ : $g \in G\}$ with degrees $d_{g}:=\operatorname{deg}\left(\eta_{g}\right)$

s.t. $A_{g} \simeq A_{g^{-1}}$. E.g. in the cohomology of fixed point sets $d_{g}$ is given by the dimension and for the Jacobian Frobenius manifolds (see the next section) $d_{g}$ fixed by the degree of $\operatorname{Hess}\left(f_{g}\right)=\rho_{g}$. Furthermore, the reconstructed $\left\{\left.\eta\right|_{\left(A_{g} \otimes A_{g^{-1}}\right.}, g \in G\right\}$ have degree $d_{g}=d_{g^{-1}}$.

For a $G$-twisted FA the degrees all need to be equal to $d:=d_{e}$. To achieve this, one can shift the grading in each $A_{g}$ by $s_{g}$. This 
amounts to assigning degree $s_{g}$ to $1_{g}$. This is the only freedom, since the multiplication should be degree-preserving and all $A_{g}$ are cyclic.

Set

$s_{g}^{+}:=s_{g}+s_{g^{-1}}$

$s_{g}^{-}:=s_{g}-s_{g^{-1}}$

Then $s_{g}^{+}:=d-d_{g}$ for grading reasons, but the shift $s^{-}$is more elusive.

4.10.2. Definition. The standard shift for a Jacobian Frobenius algebra is given by

$$
s_{g}^{+}:=d-d_{g}
$$

and

$$
\begin{array}{r}
s_{g}^{-}:=\frac{1}{2 \pi i} \operatorname{tr}(\log (g))-\operatorname{tr}\left(\log \left(g^{-1}\right)\right):=\frac{1}{2 \pi i}\left(\sum_{i} l_{i}(g)-\sum_{i} l_{i}\left(g^{-1}\right)\right) \\
=\sum_{i: l_{i} \neq 0} 2\left(\frac{1}{2 \pi i} l_{i}(g)-1\right)
\end{array}
$$

where the $l_{i}(g)$ are the logarithms of the eigenvalues of $g$ using the branch with values in $[0,2 \pi)$ i.e. cut along the positive real axis.

In this case we obtain:

$$
s_{g}=\frac{1}{2}\left(s_{g}^{+}+s_{g}^{-}\right)=\frac{1}{2}\left(d-d_{g}\right)+\sum_{i}\left(\frac{1}{2 \pi i} l_{i}(g)-\frac{1}{2}\right)
$$

4.10.3. Remark. The shift $s_{g}^{-}$is canonical in the case of quasihomogeneous singularities upon replacing the classical monodromy operator $J$ by $J g$. This will be discussed elsewhere [K2].

The degree of $\gamma_{g, g 1}$ is $s^{+}$from comparing degrees in the equation $1_{g} 1_{g^{-1}}=\gamma_{g, g 1} 1_{e}$.

4.10.4. Reconstruction for graded special $G$-Frobenius algebras. In the Reconstruction program the presence of a non-trivial grading can greatly simplify the check of the trace axiom. E.g. if $A_{[g, h]}$ has no element of degree 0 then both sides of this requirement are 0 and if $[g, h]=e$ one needs only to look at the special choices of $c$ with $\operatorname{deg}(c)=0$ which most often is just $c=1$, the identity.

4.10.5. Ramond-grading. The grading in the Ramond-sector is by the following definition

This yields

$$
\operatorname{deg}(v):=-\frac{d}{2}
$$




$$
\operatorname{deg}(\bar{\eta})=0 \text { and } \operatorname{deg}(\overline{\mathrm{o}})=\frac{d}{2}
$$

\section{JACOBIAN FrobeniUs ALGEBRAS}

\subsection{Definition. A Frobenius algebra $A$ is called Jacobian}

if it can be represented as the Milnor ring of a function $f$. I.e. if there is a function $f \in \mathcal{O}_{\mathbf{A}_{K}^{n}}$ s.t. $A=\mathcal{O}_{\mathbf{A}_{K}^{n}} / J_{f}$ where $J_{f}$ is the Jacobian ideal of $f$. And the bilinear form is given by the residue pairing. This is the form given by the the Hessian of $f: \rho=\operatorname{Hess}_{f}$.

If we write $\mathcal{O}_{\mathbf{A}_{k}^{n}}=k\left[x_{1} \ldots x_{n}\right], J_{f}$ is the ideal spanned by the $\frac{\partial f}{\partial x_{i}}$.

A realization of a Jacobian Frobenius algebra is a pair $(A, f)$ of a Jacobian Frobenius algebra and a function $f$ on some affine $k$ space $\mathbf{A}_{k}^{n}$, i.e. $f \in \mathcal{O}_{\mathbf{A}_{k}^{n}}=k\left[x_{1} \ldots x_{n}\right]$ s.t. $A=k\left[x_{1} \ldots x_{n}\right]$ and $\rho:=\operatorname{det}\left(\frac{\partial^{2} f}{\partial x_{i} \partial x_{j}}\right)$.

A small realization of a Jacobian Frobenius algebra is a realization of minimal dimension, i.e. of minimal $n$.

5.2. Definition. A natural $G$ action on a realization of a Jacobian Frobenius algebra $\left(A_{e}, f\right)$ is a linear $G$ action on $\mathbf{A}_{k}^{n}$ which leaves $f$ invariant.

Given a natural $G$ action on a realization of a Jacobian Frobenius algebra $(A, f)$ set for each $g \in G, \mathcal{O}_{g}:=\mathcal{O}_{\operatorname{Fix}_{g}\left(\mathbf{A}_{k}^{n}\right)}$. This is the ring of functions of the fixed point set of $g$ for the $G$ action on $\mathbf{A}_{k}^{n}$. These are the functions fixed by $g: \mathcal{O}_{g}=k\left[x_{1}, \ldots, x_{n}\right]^{g}$.

Denote by $J_{g}:=J_{\left.f\right|_{\mathrm{Fix}_{g}\left(\mathbf{A}_{k}^{n}\right)}}$ the Jacobian ideal of $f$ restricted to the fixed point set of $g$.

Define

$$
A_{g}:=\mathcal{O}_{g} / J_{g}
$$

The $A_{g}$ will be called twisted sectors for $g \neq 1$. Notice that each $A_{g}$ is a Jacobian Frobenius algebra with the natural realization given by $\left(A_{g},\left.f\right|_{\text {Fix }_{g}}\right)$. In particular, it comes equipped with an invariant bilinear form $\tilde{\eta}_{g}$ defined by the element $\operatorname{Hess}\left(\left.f\right|_{\text {Fix }_{g}}\right)$.

For $g=1$ the definition of $A_{e}$ is just the realization of the original Frobenius algebra, which we also call the untwisted sector.

Notice there is a restriction morphism $r_{g}: A_{e} \rightarrow A_{g}$ given by $a \mapsto$ $\left.a\right|_{\text {Fix }_{g}} \bmod J_{g}$.

Denote $r_{g}(1)$ by $1_{g}$. This is a non-zero element of $A_{g}$ since the action was linear. Furthermore it generates $A_{g}$ as a cyclic $A_{e}$ module.

The set $\operatorname{Fix}_{g} \mathbf{A}_{k}^{n}$ is a linear subspace. Let $I_{g}$ be the vanishing ideal of this space. 
We obtain a sequence

$$
0 \rightarrow I_{g} \rightarrow A_{e} \stackrel{r_{g}}{\rightarrow} A_{g} \rightarrow 0
$$

Let $i_{a}$ be any splitting of this sequence induced by the inclusion: $\hat{i}_{g}: \mathcal{O}_{g} \rightarrow \mathcal{O}_{e}$ which descends due to the invariance of $f$.

In coordinates, we have the following description. Let $\operatorname{Fix}_{g} \mathbf{A}_{k}^{n}$ be given by equations $x_{i}=0: i \in N_{g}$ for some index set $N_{g}$.

Choosing complementary generators $x_{j}: j \in T_{g}$ we have $\mathcal{O}_{g}=k\left[x_{j}\right.$ : $\left.j \in T_{g}\right]$ and $\mathcal{O}_{e}=k\left[x_{j}, x_{i}: j \in T_{g}, i \in N_{g}\right]$. Then $I_{g}=\left(x_{i}: i \in N_{g}\right)_{\mathcal{O}_{e}}$ the ideal in $\mathcal{O}_{e}$ generated by the $x_{i}$ and $\mathcal{O}_{e}=I_{g} \oplus i_{g}\left(A_{g}\right)$ using the splitting $i_{g}$ coming from the natural inclusion $\hat{i}_{g}: k\left[x_{j}: j \in T_{g}\right] \rightarrow$ $k\left[x_{j}, x_{i}: j \in T_{g}, i \in N_{g}\right]$. We also define the projections

$$
\pi_{g}: A_{1} \rightarrow A_{g} ; \pi_{g}=i_{g} \circ r_{g}
$$

which in coordinates are given by $\left.f \mapsto f\right|_{x_{j}=0: j \in N_{g}}$ Let

$$
A:=\bigoplus_{g \in G} A_{g}
$$

where the sum is a sum of $A_{e}$ modules.

5.3. Definition. A discrete torsion for a group $G$ is a map from commuting pairs $(g, h) \in G \times G:[g, h]=e$ to $k^{*}$ with the properties:

$$
\epsilon(g, h)=\epsilon\left(h^{-1}, g\right) \quad \epsilon(g, g)=1 \quad \epsilon\left(g_{1} g_{2}, h\right)=\epsilon\left(g_{1}, h\right) \epsilon\left(g_{2}, h\right)
$$

5.4. Theorem. Given a natural $G$ action on a realization of a Jacobian Frobenius algebra $\left(A_{e}, f\right)$ with a quasi-homogeneous function $f$ of degree $d$ and type $\mathbf{q}=\left(q_{1}, \ldots, q_{n}\right)$ together with the natural choice of splittings $i_{g}$ the possible structures of special $G$ twisted Frobenius algebra on the $A_{e}$ module $A:=\bigoplus_{g \in G} A_{g}$ are in 1-1 correspondence with elements of $\bar{Z}^{2}(G, A)$, where $\bar{Z}^{2}$ are $G$ graded cocycles and a compatible non-abelian two cocycle $\varphi$ with values in $k^{*}$, which define a choice of discrete torsion.

Proof. By the reconstruction Theorem the structure of a special $G$ twisted Frobenius algebra on the given data $A_{g}, \eta_{g}, i_{g}$ is determined by:

- A graded $G 2$-cocycle with values in $A_{e}$ and a compatible nonabelian $G 2$-cocycle with values in $k^{*}$

- A group homomorphism $\chi: G \rightarrow k^{*}$

subject to the conditions:

i) $\eta_{e}\left(\varphi_{g}(a), \varphi_{g}(b)\right)=\chi_{g}^{-2} \eta_{e}(a, b)$

ii) $\eta_{g}\left(a_{g}, b_{g}\right)=\eta\left(i_{g}\left(a_{g} b_{g}\right) \gamma_{g g^{-1}}, 1\right)$ 
iii) For any $c \in A_{[g h]}$ :

$$
\chi_{h} \mathrm{~S} \operatorname{Tr}\left(\left.l_{c} \varphi_{h}\right|_{A_{g}}\right)=\chi_{g^{-1}} \mathrm{~S} \operatorname{Tr}\left(\left.\varphi_{g^{-1}} l_{c}\right|_{A_{h}}\right)
$$

where we can restrict to the $c$ with $\operatorname{deg}(c)=0$.

The $\chi_{g}$ are fixed by the equation

$$
(-1)^{\tilde{g}} \operatorname{dim}\left(A_{g}\right)=\mathrm{S} \operatorname{Tr}\left(\left.i d\right|_{A_{g^{-1}}}\right)=\chi_{g} \mathrm{~S} \operatorname{Tr}\left(\left.\varphi_{g}\right|_{A_{e}}\right)=\chi_{g} \operatorname{Tr}\left(\left.\varphi_{g}\right|_{A_{e}}\right)
$$

To calculate the trace on the RHS, we use the character function for a morphism $g$ of degree 0 on graded module $V=\oplus_{n} V_{n}$ :

$$
\chi_{V_{n}}(g, z):=\sum_{n, \mu} \mu \operatorname{dim}\left(V_{\mu, n}\right) z^{n}
$$

where $V_{\mu, n}$ is the Eigenspace of Eigenvalue $\mu$ on the space $V_{n}$. We will use the grading induced by the quasi-homogeneity. I.e. let $N$ be such that $q_{i}=Q_{i} / N$ with $Q_{i} \in \mathbf{N}$ and $N$ s.t. $|G| \mid N$. Then a monomial has degree $n$ if its quasi homogeneous degree is $n / N$. This is the natural grading for the quasi-homogeneous map $\operatorname{grad}(f)$. Notice that since $g$ commutes with $f$ it preserves the grading. It is clear that this character behaves multiplicatively under concatenations of quasi-homogeneous functions. Therefore by applying Arnold's method, we can pass to a cover of $k^{n}$ with the projection map $T: T\left(x_{1}, \ldots, x_{n}\right)=\left(x^{q_{1}}, \ldots, x^{q_{n}}\right)$ and calculate the character for $T$ and for $\operatorname{grad}(f) \circ T$. Then repeating the argument in a simultaneous Eigenbasis of $g$ and the grading of $[\mathrm{A}]$, we obtain:

$$
\chi_{A_{e}}(g, z)=\prod_{i=1}^{n} \frac{\left(\tilde{\mu}_{i} z\right)^{N-Q_{i}}-1}{\left(\tilde{\mu}_{i} z\right)^{Q_{i}}-1}
$$

where the $\tilde{\mu}_{i}$ are the Eigenvalues of some lift of the action of $g$ i.e. $\tilde{\mu}_{i}^{Q_{i}}=\mu_{i}$. Notice that since $|G| \mid N \tilde{\mu}_{i}^{N}=1$, so that in the limit of $z \rightarrow 1$, we obtain:

$$
\operatorname{Tr}\left(\left.\varphi_{g}\right|_{A_{e}}\right)=\prod_{i: \mu_{i} \neq 1}-\mu_{i}^{-1} \prod_{i: \mu_{i}=1} \frac{1}{q_{i}}-1=(-1)^{\left|N_{g}\right|} \operatorname{det}(g)^{-1} \operatorname{dim}\left(A_{g}\right)
$$

so that

We set

$$
\chi_{g}=(-1)^{\tilde{g}}(-1)^{\left|N_{g}\right|} \operatorname{det}(g)
$$

$$
\sigma(g):=\tilde{g}+\left|N_{g}\right| \bmod 2
$$


and call it the sign of $g$. Notice that $\sigma \in \operatorname{Hom}(G, \mathbf{Z} / 2 \mathbf{Z})$ since both det and $\chi$ are characters. Also notice that a choice of sign corresponds to a choice of parity and vice-versa. Thus we obtain

$$
\chi_{g}=(-1)^{\sigma(g)} \operatorname{det}(g)
$$

This ensures condition i). Since for $\rho=\operatorname{Hess}(f)$ :

$$
\varphi_{g}(\rho)=\operatorname{det}(g)^{-2} \rho
$$

We rescale $\eta_{g}$ by $\left((-1)^{\tilde{g}} \chi_{g}\right)^{1 / 2} \eta_{g}$ where we choose to cut the plane along the negative real axis. This uniquely defines a square root unless $(-1)^{\tilde{g}} \chi_{g}=-1$. In the case that $g^{2} \neq e$ we can choose roots $i$ and $-i$ for $g$ respectively $g^{-1}$. The only case that has no solution would be the case of $g^{2}=e$ and $(-1)^{\tilde{g}} \chi_{g}=-1$, but this means that either $\chi_{g}=-1$ and $\tilde{g}=1$ or $\chi_{g}=-1$ and $\tilde{g}=0$ which cannot happen, since in this case $(-1)^{\left|N_{g}\right|}=\operatorname{det}(g)$ and $(-1)^{\tilde{g}}(-1)^{\left|N_{g}\right|} \operatorname{det}(g)=\chi_{g}$. Then $\eta_{g}$ will satisfy the reconstruction conditions.

Finally, we need to check the validity of iii). Notice that since the multiplication is graded the traces are 0 unless $\operatorname{deg}\left(\gamma_{c}\right)=0$ so that we can assume that $\gamma_{c}=1$. In this case, we have to show:

$$
\chi_{h} \operatorname{STr}\left(\left.\varphi_{h}\right|_{A_{g}}\right)=\chi_{g^{-1}} \mathrm{~S} \operatorname{Tr}\left(\left.\varphi_{g^{-1}}\right|_{A_{h}}\right)
$$

Let $x_{i}$ be a basis of $A_{e}$ in which $g$ is diagonal. Then we have to compute the trace of the action of $h$ on the sub-algebra generated by the $x_{i}$ with eigenvalue 1 under the action of $g$. This is just the truncated version of the calculation above, so diagonalizing $h$ on $k\left[x_{i}: i \in T_{g}\right]$ we find using the same characteristic functions and rationale as before:

$$
\begin{aligned}
& \chi_{h} \operatorname{STr}\left(\left.\varphi_{h}\right|_{A_{g}}\right)=\chi_{h} \varphi_{h, g}(-1)^{\tilde{g}} \operatorname{Tr}\left(\left.\varphi_{h}\right|_{i_{g}\left(A_{g}\right)}\right) \\
= & \chi_{h} \varphi_{h, g}(-1)^{\tilde{g}} \prod_{j: \nu_{j} \neq 1}-\nu_{j}^{-1} \prod_{j: \nu_{j}=1} \frac{1}{q_{j}}-1 \\
= & \chi_{h} \varphi_{h, g}(-1)^{\tilde{g}}(-1)^{\left|T_{g}\right|}(-1)^{\left|T_{g} \cap T_{h}\right|} \operatorname{det}\left(\left.h\right|_{T_{g}}\right)^{-1} \operatorname{dim}\left(i_{g}\left(A_{g}\right) \cap i_{h}\left(A_{h}\right)\right) \\
= & \varphi_{h, g}(-1)^{\sigma(g)}(-1)^{\sigma(h)} \operatorname{det}(h) \operatorname{det}\left(\left.h\right|_{T_{g}}\right)^{-1}(-1)^{N}(-1)^{\left|T_{g} \cap T_{h}\right|} \operatorname{dim}\left(i_{g}\left(A_{g}\right) \cap i_{h}\left(A_{h}\right)\right) \\
= & \varphi_{h, g}(-1)^{\sigma(h g)} \operatorname{det}\left(\left.h\right|_{N_{g}}\right)(-1)^{\left|T_{g} \cap T_{h}\right|+N} \operatorname{dim}\left(i_{g}\left(A_{g}\right) \cap i_{h}\left(A_{h}\right)\right) \\
= & \epsilon(h, g) T(h, g)
\end{aligned}
$$

where $\nu_{j}$ are the Eigenvalues of $h$ on $i_{g}\left(A_{g}\right)$ and

$$
\epsilon(g, h)=\varphi_{g, h}(-1)^{\sigma(g) \sigma(h)} \operatorname{det}\left(\left.g\right|_{N_{h}}\right)
$$

and we set $\operatorname{det}\left(\left.g\right|_{N_{h}}\right):=\operatorname{det}(g) \operatorname{det}^{-1}\left(\left.g\right|_{T_{h}}\right)$ if $[g, h] \neq e$.

$$
T(h, g)=(-1)^{\sigma(g) \sigma(h)}(-1)^{\sigma(g)+\sigma(h)}(-1)^{\left|T_{g} \cap T_{h}\right|+N} \operatorname{dim}\left(i_{g}\left(A_{g}\right) \cap i_{h}\left(A_{h}\right)\right)
$$


we have that

$$
T(h, g)=T(g, h)=T\left(g^{-1}, h\right)
$$

Notice also that if $[g, h]=e$

$$
\epsilon(g h, k)=\epsilon(g, k) \epsilon(h, k)
$$

and

$$
T(g h, h)=T(h g, h)=T(g, h)
$$

On the other hand

$$
\begin{aligned}
& \operatorname{STr}\left(\left.\varphi_{g^{-1}}\right|_{A_{h}}\right)=\epsilon\left(g^{-1}, h\right)(-1)^{\sigma\left(g^{-1} h\right)}(-1)^{\left|T_{g} \cap T_{h}\right|+N} \operatorname{dim}\left(i_{g}\left(A_{g}\right) \cap i_{h}\left(A_{h}\right)\right) \\
= & \varphi_{h, g}(-1)^{\sigma\left(g^{-1} h\right)} \operatorname{det}\left(\left.g^{-1}\right|_{N_{h}}\right)(-1)^{\tilde{g} \tilde{h}}(-1)^{\left|T_{g} \cap T_{h}\right|} \operatorname{dim}\left(i_{g}\left(A_{g}\right) \cap i_{h}\left(A_{h}\right)\right) \\
= & \epsilon\left(g^{-1}, h\right) T\left(g^{-1}, h\right)
\end{aligned}
$$

where $\mu_{j}$ are the Eigenvalues of $g^{-1}$ on $i_{h}\left(A_{h}\right)$.

Finally we see that the $\varphi$ are determined by the $\epsilon(g, h)$ which have to satisfy

$$
\epsilon(g, h)=\epsilon\left(h^{-1}, g\right) \quad \epsilon(g, g)=1 \quad \epsilon\left(g_{1} g_{2}, h\right)=\epsilon\left(g_{1}, h\right) \epsilon\left(g_{2}, h\right)
$$

which means that the choices of the projective factors for the $G$-action correspond in a 1-1 fashion to a choice of discrete torsion.

\subsubsection{Remarks.}

1) For a section independent cocycle $\gamma$ we have that

$$
\eta\left(\gamma_{g, g^{-1}}, I_{g}\right)=0 \text { and } \eta\left(\gamma_{g, g^{-1}}, i_{g}\left(\rho_{g}\right)\right)=1
$$

where $\rho_{g}$ is the defining element of $\eta_{g}$. Thus $\gamma_{g, g^{-1}}$ is uniquely defined as $\gamma_{g, g^{-1}}=\check{r}_{g}\left(1_{g}\right)$.

where ${ }^{\sim}$ is the dual linear map in the sense of vector-spaces with non-degenerate bilinear forms.

Furthermore if $\gamma_{g, h}=\gamma_{h^{-1}, g^{-1}}=1$ then

$$
\gamma_{g h,(g h)^{-1}}=\gamma_{g, g^{-1}} \gamma_{h, h^{-1}}
$$

2) If $\forall g, h, \in G: J_{h}=J_{g}$ any choice of cocycle $\gamma$ with values in $k^{*}$ will give a special G-twisted FA. There is only one choice of compatible non-abelian cocycle $\varphi$.

3) For any other solution $\gamma$ by (4.9), we must have $\gamma_{g, h}=0$ if $[g, h]=e$ unless $\varphi_{g, h} \varphi_{h, g}=1$ or in other words

$$
\left.\operatorname{det}\left(\left.g\right|_{N_{h}}\right) \operatorname{det}(h)\right|_{N_{g}}=1
$$

since $\epsilon(g, h)=\epsilon\left(h^{-1}, g\right)=\epsilon(h, g)^{-1}$ 
Proof. For 1) notice that

$$
\eta\left(\gamma_{g, g^{-1}}, I_{g}\right)=\eta\left(\gamma_{g, g^{-1}} I_{g}, 1\right)=0
$$

and

$$
\eta\left(\gamma_{g, g^{-1}}, i_{g}\left(\rho_{g}\right)\right)=\eta_{g}\left(1, \rho_{g}\right)=1
$$

regardless of the choice of $i_{g}$, by the previous equation. For the uniqueness notice that in the graded case the degree $d-\operatorname{deg}\left(\gamma_{g, g^{-1}}\right)$ piece of $A$ is the direct sum of $i_{g}\left(\rho_{g}\right)$ and the degree $d-\operatorname{deg}\left(\gamma_{g, g^{-1}}\right)$ piece of $I_{g}$.

Furthermore we have that

$$
\gamma_{g h,(g h)^{-1}}=\gamma_{g, h} \gamma_{g h,(g h)^{-1}}=\gamma_{g, g^{-1}} \gamma_{h, h^{-1} g^{-1}}=\gamma_{g, g^{-1}} \gamma_{h, h^{-1}}
$$

since

$$
\pi_{g^{-1}}\left(\gamma_{h, h^{-1} g^{-1}}\right)=\pi_{g^{-1}}\left(\gamma_{h^{-1}, g^{-1}} g_{h, h^{-1} g^{-1}}\right)=\pi_{g^{-1}}\left(\gamma_{h, h^{-}} \gamma_{e, g^{-1}}\right)
$$

and $I_{g^{-1}} \gamma_{g, g^{-1}}=0$. This is useful for calculations; another proof is: $\forall a \in A_{e}$

$$
\eta\left(a, \check{r}_{g}\left(1_{g}\right)\right)=\eta_{g}\left(r_{g}(a), 1_{g}\right)=\eta\left(\pi_{g}(a), \gamma_{g, g^{-1}}\right)=\eta\left(a, \gamma_{g, g^{-1}}\right)
$$

For 2) notice that all $\pi_{h}\left(\gamma_{g, g^{-1}}\right) \neq 0$. Thus by 4.10 the $\gamma_{g, h} \neq 0$ and furthermore since $r_{g}=i d$, we see that the $\gamma_{g, h} \in k^{*}$.

For 3) we use the compatibility twice. If $[g, h]=e$ : $\gamma_{g, h}=\varphi_{g, h} \gamma_{h, g}=\varphi_{g, h} \varphi_{h, g} \gamma_{g, h}$

Now since by assumption $\gamma_{g, h} \neq 0$ we get the desired result.

6. Mirror CONStruction For SPECIAL $G$-Frobenius ALGEBras

6.1. Double grading. We consider Frobenius algebras with grading in some abelian group $I$.

$$
A=\bigoplus_{i \in I} A_{i}
$$

This grading can be trivially extended to a double grading with values in $I \times I$ in two ways

$$
A^{c c}=\bigoplus_{i \in I} A_{i, i}
$$

and

$$
A^{a c}=\bigoplus_{i \in I} A_{i,-i}
$$

corresponding to the diagonal $\Delta: I \rightarrow I \times I$ and $(i d,-) \circ \Delta: I \rightarrow$ $I \times I$. We call bi-graded Frobenius algebras of this form of $(c, c)-$ type and of $(a, c)$-type, respectively. In the language of Euler fields we consider the field $(E, \bar{E})=(E, E)$ or $(E, \bar{E})=(E,-E)$. 
These gradings become interesting for special $G$-Frobenius algebras, since in that case the shifts will produce a possible non-diagonal grading.

6.2. Definition. Given a graded special $G$-Frobenius algebra we assign the following bi-degrees to $1_{g}$

$$
(E, \bar{E})\left(1_{g}\right):=\left(s_{g}, s_{g}^{-1}\right)
$$

It is clear that $A_{e}$ is of $(c, c)$-type. $A$ is however only of $(c, c)$ type if $s_{g}=s_{g}^{-1}$.

Furthermore for the Ramond-space of $A$ we assign the following bidegree to $v$

$$
(E, \bar{E})(v):=\left(-\frac{d}{2},-\frac{d}{2}\right)
$$

6.3. Euler-twist (spectral flow). In this section, we consider a graded special $G$-Frobenius algebra and construct a new vector-space from it. We denote the grading operator by $E$ :

6.3.1. Definition. The twist-operator $j$ for an Euler-field $E$ is

$$
j:=\exp (2 \pi i E)
$$

We denote the group generated by $j$ by $J$.

We call a special $G$-Frobenius Euler if there is a special $\tilde{G}$-Frobenius algebra $\tilde{A}$ of which $A$ is a subalgebra where $\tilde{G}$ is a group that has $G$ and $J$ as subgroups.

6.3.2. Definition. The dual $\check{A}$ to an Euler special $G$-Frobenius algebra $A$ of $(c, c)$-type is the vector space

$$
\check{A}:=\bigoplus_{g \in G} \check{A}_{g}:=\bigoplus_{g \in G} V_{g j^{-1}}
$$

with the $A_{e}$ and $G$-module structure determined by $\tau_{j}^{g}: \check{A}_{g} \simeq V_{g j^{-1}}$ together with the bi-grading

$$
(E, \bar{E})\left(\check{1}_{g}\right):=\left(s_{g j^{-1}}-d, s_{g j 1}\right)
$$

where $\check{1}_{g}$ denote the generator of $\check{A}_{g}$ as $A_{e}$-module and the bi-linear form

$$
\check{\eta}:=\tau_{j}^{*}(\bar{\eta})
$$

where $\tau_{j}:=\oplus_{g \in G} \tau_{j}^{g}$ and $V$ and $\bar{\eta}$ refer to the Ramond-space of $A$. 


\section{EXPLiCIT EXAMPLES}

7.1. Self duality of $A_{n}$. We consider the example of the Jacobian Frobenius Algebra associated to the function $z^{n+1}$

$$
A_{n}:=\mathbf{C}[z] /\left(z^{n}\right)
$$

together with the induced multiplication and the Grothendieck residue. Explicitly:

the form

$$
z^{i} z^{j}= \begin{cases}z^{i+j} & \text { if } i+j \leq n \\ 0 & \text { else }\end{cases}
$$

and the grading:

$$
\eta\left(z^{i}, z^{j}\right)=\delta_{i, n-1-j}
$$

$$
E\left(z^{i}\right):=\frac{i}{n+1}
$$

which means $\rho=z^{n-1}$ and $d=\frac{n-1}{n+1}$.

We consider just the group $J \simeq \mathbf{Z} /(n+1) \mathbf{Z}$ with the generator $j$ acting on $z$ by multiplication with $\zeta_{n+1}:=\exp \left(2 \pi i \frac{1}{n+1}\right)$. We have

$$
\text { Fix }_{j^{i}} \begin{cases}\mathbf{C} & \text { if } i=0 \\ 0 & \text { else }\end{cases}
$$

and thus

$$
A_{j^{i}} \begin{cases}A_{n} & \text { if } i=0 \\ 1_{j^{i}} & \text { else }\end{cases}
$$

Furthermore we have the following grading;

$$
(E, \bar{E})\left(1_{j^{i}}\right)= \begin{cases}(0,0) & \text { for } i=0 \\ \left(\frac{k-1}{n+1}, \frac{n-k}{n+1}\right) & \text { else }\end{cases}
$$

which means $\rho_{j^{i}}=1_{j^{i}}$ and $d_{j^{i}}=0$.

Using the reconstruction Theorem we have to find a cocycle $\gamma$ and a compatible action $\varphi$. There is no problem for the metric since always $\left|N_{g}\right|=1$ and if $n+1$ is even $\operatorname{det}\left(\zeta^{\frac{n+1}{2}}\right)=-1$. Since the group $J$ is cyclic there is no freedom of choice for $\epsilon$ and just two choices of parity are possible corresponding to $j \mapsto \pm 1$.

From the general considerations we know $\gamma_{j^{i}, j^{n-1-i}} \in A_{e}$ and $\operatorname{deg}\left(\gamma_{j^{i}, j^{n-1-i}}\right)=$ $d-d_{j^{i}}=\frac{n-1}{n+1}$ which yields

$$
\gamma_{j^{i}, j^{n-1-i}}=\left((-1)^{\tilde{j} i} \zeta^{i}\right)^{1 / 2} \rho=\left((-1)^{\tilde{j}} \zeta\right)^{i / 2} z^{n-1}
$$


for the other $\gamma$ notice that $\operatorname{deg}\left(1_{j^{i}}\right)+\operatorname{deg}\left(1_{j^{k}}\right)=\frac{i+k-2}{n+1}$ while $\operatorname{deg}\left(1_{j^{i+j}}\right)=$ $\frac{i+k-1}{n+1}$ if $i+k \neq n+1$, but there is no element of degree $\frac{1}{n+1}$ in $A_{j^{i+k}}$ for $i+k \neq n+1$.

Hence

$$
\gamma_{j^{i}, j^{k}}= \begin{cases}\left((-1)^{\tilde{j}} \zeta\right)^{i / 2} z^{n-1} & \text { for } i+j=n+1 \\ 0 & \text { else }\end{cases}
$$

this means that

$$
\varphi_{j^{i}, j^{k}}=(-1)^{\tilde{j}^{\tilde{j}^{k}}} \zeta^{-i}
$$

Therefore the $G$-invariants $A^{G}=A_{1}$ are generated by the identity 1.

The Ramond grading of this algebra is

$$
(E, \bar{E})\left(1_{j^{i}} v\right)= \begin{cases}(0,0) & \text { for } i=0 \\ \left(\frac{k}{n+1}-\frac{1}{2},-\frac{k}{n+1}+\frac{1}{2}\right) & \text { else }\end{cases}
$$

Since $j \in G$ the special $G$-Frobenius algebra is Euler and the dual is defined, moreover $G=J$ so that the vector-space structures of $A$ and $\check{A}$ coincide. The grading is given by:

$$
(E, \bar{E})\left(1_{j^{i}} v\right)= \begin{cases}(0,0) & \text { for } i=0 \\ \left(-\frac{k-(n+1)}{n+1}-\frac{1}{2}, \frac{n+1-k}{n+1}+\frac{1}{2}\right) & \text { else }\end{cases}
$$

The $G$-action is given by

$$
\check{\varphi}_{j^{i}, j^{k}}= \begin{cases}(-1)^{\tilde{j}^{i} \tilde{j}^{k}} \zeta^{i} & \text { for } k=1 \\ (-1)^{\tilde{j}^{i} \tilde{j}^{k}} & \text { else }\end{cases}
$$

This $G$-action leaves all even sectors $\check{A}_{j^{i}}$ invariant except for $i=1$ if $\tilde{j}=1$. Thus with the choice of all even sectors we have as $G$-modules $\check{A} \simeq A_{n}$ where more explicitly $\check{1}_{j^{i}} \mapsto z^{n+1-k}: k=2, \ldots n$ and $\check{1}_{0} \mapsto 1$.

Notice that this $A_{n}$ is of $(a, c)$-type however. Also since $J=G$ the form $\bar{\eta}$ pulls back and gives a non-degenerate form on $\check{A}^{G}$, which is the usual form on $A_{n}$. Furthermore the usual multiplication on $A_{n}$ is compatible with everything so that we can say that $A_{n}$ is self-dual under this operation.

7.2. $D_{n}$ from a special $\mathbf{Z} / 2 \mathbf{Z}$-Frobenius algebra based on $A_{2 n-3}$. In this section, we show how to get $D_{n}$ from a special $\mathbf{Z} / 2 \mathbf{Z}$-Frobenius algebra based on $A_{2 n-3}$. The function for the Frobenius algebra $A_{2 n-3}$ is $z^{2 n-2}$. Since this is an even function, $\mathbf{Z} / 2 \mathbf{Z}$ acting via $z \mapsto-z$ is a symmetry. There are two sectors, the untwisted and the twisted sector 
containing the element $1_{-1}$ with degree 0 . The multiplication is fixed by $\operatorname{deg}\left(\gamma_{-1,-1}\right)=\frac{2 n-4}{2 n-2}$ thus

$$
\gamma_{-1,-1}=z^{2(n-2)}
$$

again the group is cyclic so the $G$-action only depends on the choice of parity of the -1 -sector.

In the untwisted sector we have $A_{e}^{\mathbf{Z} / 2 \mathbf{Z}}=\left\langle 1, z^{2}, \ldots, z^{2(n-1)}\right\rangle \simeq A_{n-1}$ and the action of -1 on $1_{-1}$ is given by

$$
\varphi_{-1,-1}=(-1)^{\widetilde{-1} \widetilde{-1}+1}
$$

so that if $\sigma(-1)=1$

$$
A_{2 n-3, \mathbf{Z} / 2 \mathbf{Z}}^{\mathbf{Z} / 2 \mathbf{Z}} \simeq D_{n}
$$

and if $\sigma(-1)=0$ we just obtain the invariants of the untwisted sector which are isomorphic to $A_{n-1}$.

The untwisted sector is given by the singularity $A_{n-1}$ as expected upon the transformation $u=z^{2}$. Notice that the invariants of the Ramond sector yield the singularity $A_{n-2}$ as expected from [Wa]. These are of the form $u^{i} d u$ or $z^{2 i+1} d z$ with $i=0, \ldots, n-3$.

7.3. Point $\bmod G$. In the theory of Jacobian Frobenius algebras there is the notion of a point played by a Morse singularity $z_{1}^{2}+\cdots+z_{n}^{2}$. Any finite subgroup $G \subset O(n, k)$ leaves this point invariant.

The $G$-twisted algebra after possibly stabilizing is the following.

$$
A=\bigoplus_{g \in G} k 1_{g}
$$

And the grading is $\operatorname{deg}\left(1_{g}\right)=\left(\frac{1}{2} s_{g}^{-}, \frac{1}{2} s_{g}^{-}\right)$, since $d=d_{g}=1$.

Using 4.10 it follows that the $\psi$ cannot vanish, thus fixing $\phi$ and $\epsilon$, so that the possibilities are enumerated by the graded cocycles. The compatibility equations hold automatically.

SPECIAL CASE.

If we assume that $G \subset O(n, \mathbf{C})$ and that $s_{g}^{-}=0$ (i.e. $\sum_{i: l_{i} \neq 0} \frac{1}{2 \pi i} l_{i}=$ $\left.\frac{\left|N_{g}\right|}{2} \in \mathbf{N}\right)$, then we the cocycles lie in $H^{2}\left(G, k^{*}\right)$ and the possible algebra structures are those of twisted group algebras.

7.3.1. Point $\bmod \mathbf{Z} / n \mathbf{Z}$. By the above analysis we realize $\mathbf{Z} / n \mathbf{Z}$ as the sub-group of rotations of order $n$ in $\mathbf{C}$. We have that $s_{g}^{-}=0$ and thus we can choose the full cocycle making $A$ into $A_{n}$, multiplicatively, with trivial grading and trivial $G$-action if one chooses all even sectors. The metric, however, will not be consistent with $A_{n}$. The identity pairs with itself for instance. Dualizing $A$, we obtain the following space

$$
\check{A}=\oplus_{j^{i}} A_{j^{i}-1}
$$


with again a trivial $(E, \bar{E})$ grading. Choosing the generator $\tilde{j}:=j^{-1}$ for $J$, the metric reads

$$
\tilde{\eta}\left(\tilde{1}_{\tilde{j}^{i}}, \tilde{1}_{\tilde{j}^{k}}\right)= \begin{cases}1 & \text { if } i+j=n-1 \\ 1 & i=j=n \\ 0 & \text { else }\end{cases}
$$

This metric is compatible with the following multiplication:

$$
\tilde{1}_{\tilde{j}^{i}} \circ \tilde{1}_{\tilde{j}^{k}}= \begin{cases}\tilde{1}_{\tilde{j}^{i+k}} & \text { if } i+j \leq n-1 \\ \tilde{1}_{\tilde{j}^{n-1}} & i=j=n \\ 0 & \text { else }\end{cases}
$$

The structure of this algebra is the one obtained in the $r$-spin curve picture of the $A_{n}$-model. Which in mirror symmetry parlance the Amodel version of the theory. By our mirror symmetry we see that we obtain this $\mathrm{A}$-model by orbifolding the Landau-Ginzburg $\mathrm{B}$-model pair $\left(A_{n}, A_{1}\right)$ above by $\mathbf{Z} / n \mathbf{Z}$. We previous did this by regarding the left side of the pair and the above calculation is for

the right side of the pair. Thus we see that the appearance of the extra basis element can be seen as natural from this point of view. Answering the question of its emergence in [W].

\section{REFERENCES}

[A] M. Atiyah. Topological quantum field theories. Inst. Hautes Études Sci. Publ. Math. No. 68 (1988), 175-186.

[CR] W. Chen, Y. Ruan. Orbifold Quantum Cohomology. Preprint.

[Dij] R. Dijkgraaf. A geometrical approach to two-dimensional conformal field theory Ph. D. Thesis, Utrecht (1989).

[Du] B. Dubrovin. Geometry of 2D topological field theories. Integrable systems and quantum groups (Montecatini Terme, 1993), 120-348, Lecture Notes in Math., 1620, Springer, Berlin, 1996.

[DVVV] R. Dijkgraaf, C. Vafa, E. Verlinde, H. Verlinde. The operator algebra of orbifold models. Comm. Math. Phys. 123 (1989), 485-526.

[DW] R. Dijkgraaf, E. Witten. Topological gauge theories and group cohomology. Comm. Math. Phys. 129 (1990), 393-429.

[FG] B. Fantechi and L. Goettsche. Orbifold cohomology for global quotients. Preprint.

[FQ] D. Freed, F. Quinn. Chern-Simons theory with finite gauge group. Comm. Math. Phys. 156 (1993), 435-472.

[GP] B. R. Greene, M. R. Plesser Duality in Calabi-Yau moduli space. Nuclear Phys. B 338 (1990), 15-37

[HT] A. Hatcher, W. Thurston. A presentation for the mapping class group of a closed orientable surface. Topology 19 (1980), 221-237.

[IV] K. Intriligator,C. Vafa. Landau-Ginzburg orbifolds. Nuclear Phys. B 339 (1990), 95-120 
[JKV] T. Jarvis, T. Kimura and A. Vaintrob. Moduli spaces of higher spin curves and integrable hierarchies. Compositio Math. 126 (2001).

[K1] R. Kaufmann. The tensor Product in the Theory of Frobenius manifolds. Int. J. of Math. 10 (1999) 159-206.

[K2] R. Kaufmann. Singularities and orbifold Frobenius algebras. (in preparation)

[K3] R. Kaufmann. Second quantized Frobenius algebras. Preprint. math.AG/0206137.

[K4] R. Kaufmann. The algebra of discrete torsion. Preprint. math.AG/0208081

[K5] R. Kaufmann. Discrete torsion, symmetric products and the Hilbert scheme. Preprint.

[LS] M. Lehn and C. Soerger The cup product of the Hilbert scheme for K3 surfaces. Preprint.

[St] N. Steenrod. The Topology of Fibre Bundles. Princeton Mathematical Series, vol. 14. Princeton University Press, Princeton, N. J., 1951.

[T] V. Turaev. Homotopy field theory in dimension 2 and group-algebras Preprint.

[V] C. Vafa. String vacua and orbifoldized LG models. Modern Phys. Lett. A 4 (1989), 1169-1185.

[W] E. Witten, Algebraic geometry associated with matrix models of two- dimensional gravity, Topological models in modern mathematics (Stony Brook, NY, 1991), Publish or Perish, Houston, TX (1993), 235-269.

- The N-matrix model and gauged WZW models, Nucl. Phys. B371 (1992), no. $1,191245$.

[Wa] C. T. C. Wall. A note on symmetry of singularities. Bull. London Math. Soc. 12 (1980), 169-175.

E-mail address: kaufmann@math.usc.edu

University of Southern California, Los Angeles, USA 